\title{
Molecular Architecture and Biomedical Leads of Terpenes from Red Sea Marine Invertebrates
}

\author{
Mohamed Elamir F. Hegazy 1,*, Tarik A. Mohamed ${ }^{1}$, Montaser A. Alhammady ${ }^{2}$, \\ Alaa M. Shaheen ${ }^{1}$, Eman H. Reda ${ }^{1}$, Abdelsamed I. Elshamy ${ }^{3}$, Mina Aziz ${ }^{4}$ \\ and Paul W. Paré ${ }^{4, *}$
}

1 Chemistry of Medicinal Plants Department, Center of Excellence for Advanced Sciences, National Research Centre, 33 El Bohouth st., Dokki, Giza, P.O. Box 12622, Egypt; E-Mails: tarik.nrc83@yahoo.com (T.A.M.); alaasaidalah_shaheen@yahoo.com (A.M.S.); dremanhusseinreda@gmail.com (E.H.R.)

2 National Institute of Oceanography and Fisheries, Red Sea Branch, Hurghada 84511, Egypt; E-Mail: coralreef_niof1@yahoo.com

3 Natural Compounds Chemistry Department, National Research Centre, 33 El Bohouth st. (former El Tahrir st.) Dokki, Giza, P.O. Box 12622, Egypt; E-Mail: elshamynrc@yahoo.com 4 Department of Chemistry and Biochemistry, Texas Tech University, Lubbock, TX 79409, USA; E-Mail: drmina_aziz06@yahoo.com

* Authors to whom correspondence should be addressed; E-Mails: elamir77@live.com (M.E.F.H.); Paul.Pare@ttu.edu (P.W.P.); Tel.: +20-1022900036 (M.E.F.H.); +1-806-834-0461 (P.W.P.); Fax: +20-233370931 (M.E.F.H.); +1-806-742-1289 (P.W.P.).

Academic Editor: Valeria Costantino

Received: 9 April 2015 / Accepted: 7 May 2015 / Published: 20 May 2015

\begin{abstract}
Marine invertebrates including sponges, soft coral, tunicates, mollusks and bryozoan have proved to be a prolific source of bioactive natural products. Among marine-derived metabolites, terpenoids have provided a vast array of molecular architectures. These isoprenoid-derived metabolites also exhibit highly specialized biological activities ranging from nerve regeneration to blood-sugar regulation. As a result, intense research activity has been devoted to characterizing invertebrate terpenes from both a chemical and biological standpoint. This review focuses on the chemistry and biology of terpene metabolites isolated from the Red Sea ecosystem, a unique marine biome with one of the highest levels of biodiversity and specifically rich in invertebrate species.
\end{abstract}


Keywords: terpenes; Red Sea; marine ecosystem; marine invertebrates; biomedical leads

\section{Red Sea Ecosystem}

Marine ecosystems cover nearly $70 \%$ of the earth's surface, averaging almost $4 \mathrm{~km}$ in depth and are proposed to contain over $80 \%$ of the world's plant and animal species [1]. Exact marine biodiversity is less certain since between one-third and two-thirds of marine organisms have yet to be described [2]. Worldwide there are approximately 226,000 marine eukaryotes currently reported, while close to a million total species are estimated, based on calculations by marine biologists using statistical predictions [2]. Considering that constituents from higher plants along with metabolites from terrestrial microorganisms have provided a substantial fraction of the natural-product-derived drugs currently used in Western medicine [3], the potential to vastly expand the number and diversity of natural products by mining marine eukaryotes as well as associated prokaryotes from the richly diverse Red Sea ecosystem seems attainable. In fact, just within the past quarter century, the search for new marine metabolites has resulted in the isolation of upward of 10,000 compounds [4], many of which exhibit biological activity. Despite the fact that marine biodiversity far exceeds that of terrestrial ecosystems, research of marine natural products as pharmaceutical agents, is still in its infancy. Factors that contribute to the gap between terrestrial and marine derived natural products include a paucity of ethno-medical history from marine sources as well as impediments associated with collecting, identifying and chemical analysis of marine materials [5].

Notwithstanding, a combination of new diving techniques and implementation of remotely operated pods over the last decade has facilitated the characterization of marine-derived metabolites. This review encompasses secondary metabolites derived from marine invertebrates, a largely diverse group of fixed or sessile organisms, many in a stationary form although some are capable of slow primitive movement. While invertebrates lack physical defences such as a protective shell or spines, they are often rich in defence metabolites that can be utilized to attack prey or defend a habitat.

This review focused on a class of secondary defence metabolites abundant in marine invertebrates, five-carbon isoprenoid-derived terpenes. Extensive speciation from microorganisms to mammals can be attributed, at least in part to a wide range of temperatures $\left(0\right.$ to $35^{\circ} \mathrm{C}$ in arctic waters versus hydrothermal vents, respectively), pressures (1-1000 atm.), nutrient availabilities (oligotrophic to eutrophic) and lighting conditions that exist in this marine biome [6]. The analysis will be limited to the Red Sea which is considered an epicenter for marine biodiversity with an extremely high endemic biota including over 50 genera of hermatypic coral. Indeed soft coral (Cnidaria: Anthozoa: Octocorallia), which are an important structural component of coral reef communities [7,8], are approximately $40 \%$ native to the Red Sea [6]. The Red Sea, in which extensive reef formation occurs, is arguably the world's warmest (up to $35^{\circ} \mathrm{C}$ in summer) and most saline habitat ( $c a .40 \mathrm{psu}$ in the northern Red Sea) [6]. Despite the Red Sea's size and diversity of reef-associated inhabitants (for examples, see Figure 1), marine invertebrates in this ecosystem remain poorly studied compared to other large coral reef systems around the world such as the Great Barrier Reef or the Caribbean. This 
review will cover terpenes isolated from marine invertebrates of the Red Sea (Figure 2) as well as identified biological activities for compounds reported during the time period from 1980 to 2014 .

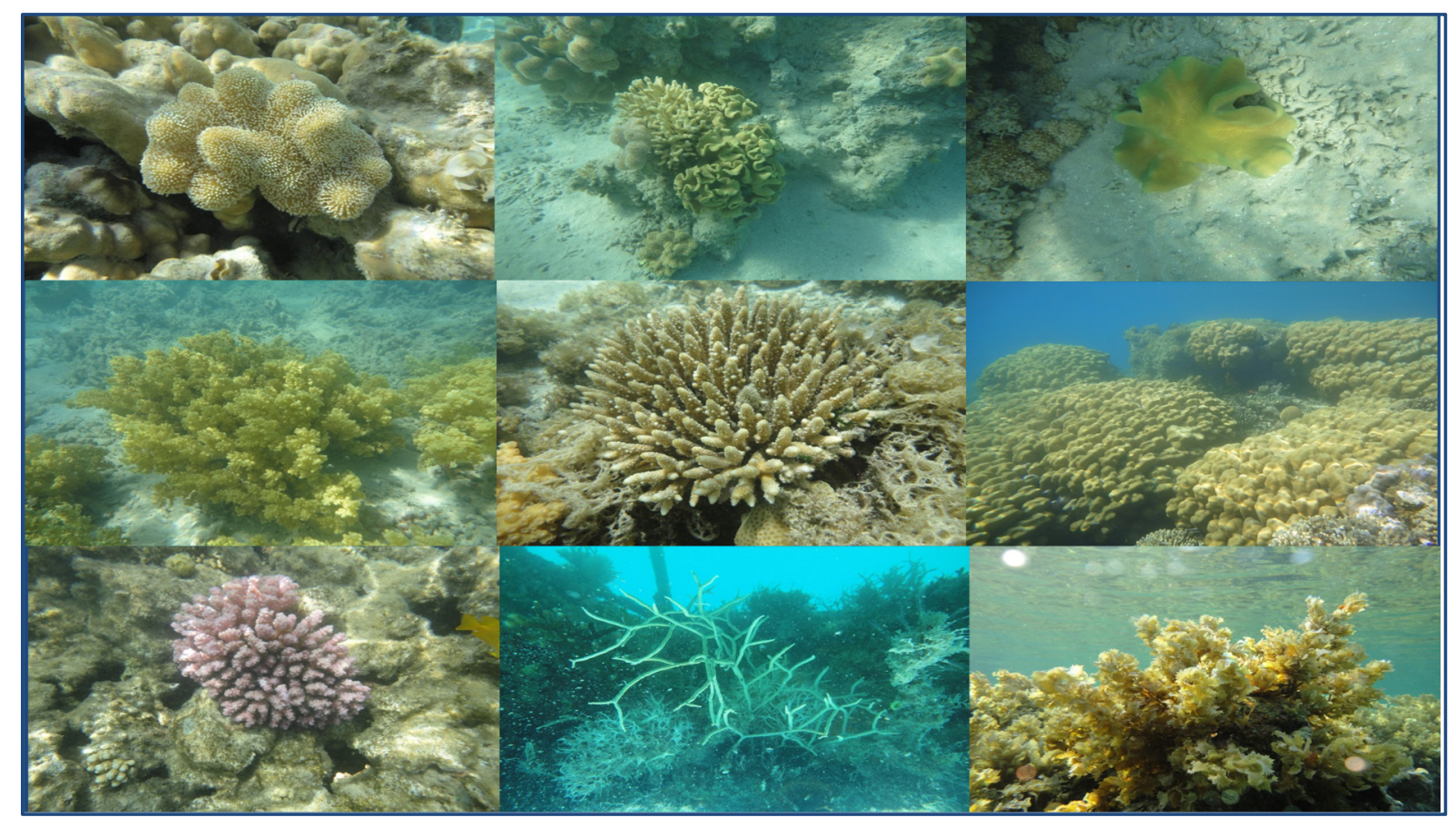

Figure 1. Samples of marine invertebrate diversity from the Red Sea including (from left to right starting at the top left corner) Sarcophyton glaucum, S. regulare, S. ehrenbergi, Nephthea molle, Acropora humilis, Porites solida, Pocillopora verrucosa, Clothraria rubrinoidis and Cystoseira trinode. Marine species exhibit greater phyta diversity than land species.

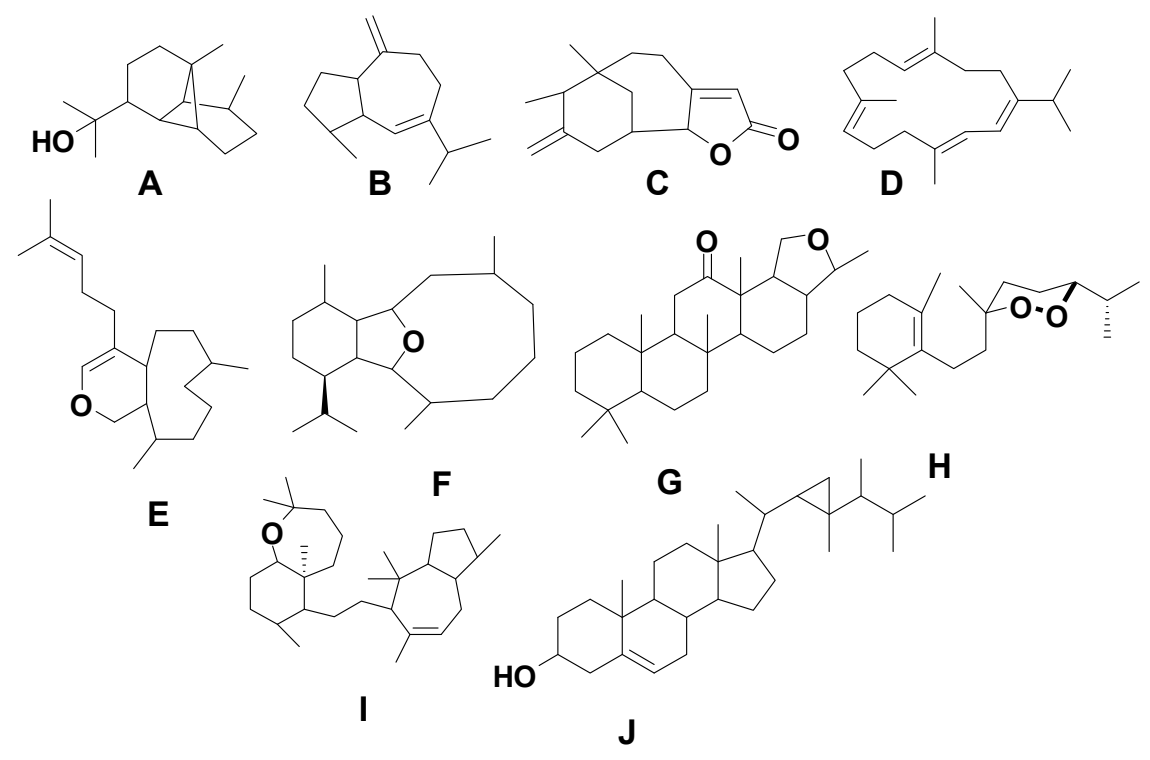

Figure 2. Terpene skeletal types including ylangene (A), aromadendrane (B), tricycle-[6,7,5]-sesquiterpene (C), cembrane (D), xeniolide and xeniaphyllane (E), eunicellin diterpene $(F)$, sesterterpene $(\mathrm{G})$, norsesterterpene $(\mathrm{H})$, triterpene $(\mathrm{I})$ and steroid (J) types. 


\section{Sesquiterpenes}

Sesquiterpenes are secondary metabolites present in many marine organisms including soft coral (e.g., Dendronephthya sp., Sinularia gardineri, Litophyton arboreum, Sarcophyton trocheliophorum, S. glaucum and Parerythropodium fulvum fulvum) [9-14], and sponges (e.g., Hyrtios sp. and Diacarnus erythraenus) [15,16].

\subsection{Ylangene-Type Sesquiterpenes}

Tricyclo-[4,6,6]-sesquiterpenes, Dendronephthol A-C (1-3) have been isolated from the soft coral Dendronephthya, family Nephtheidae (Figure 3). Cytotoxic activity was observed for $\mathbf{1}$ and $\mathbf{3}$ against the murine lymphoma L5187Y cancer cell line with ED $_{50}$ values of 8.4 and $6.8 \mu \mathrm{g} / \mathrm{mL}$, respectively [9].
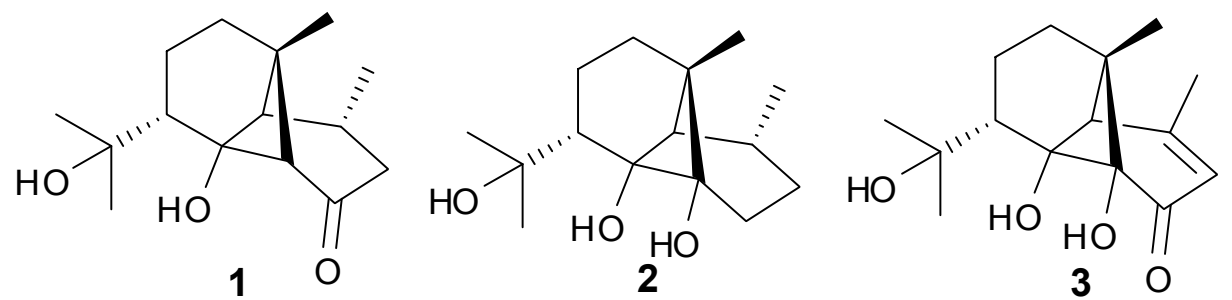

Figure 3. Representative structures of ylangene-type sesquiterpenes (1-3).

\subsection{Aromadendrane-Type Sesquiterpenes}

Bicyclico [5,7] sesquiterpenes have been isolated from several different coral with examples shown in Table 1 and Figure 4. Cytotoxicity to murine leukemia (P-388), human lung carcinoma (A-549), human colon carcinoma (HT-29), and human melanoma cells (MEL-28) [11] was observed with exposure to 4. Inhibitory activity against HIV-1 protease (PR) at an $\mathrm{IC}_{50}$ of $7 \mu \mathrm{M}$ was observed for 5 . Compounds $\mathbf{5}$ and $\mathbf{8}$ demonstrated cytostatic action with assaying HeLa cells, revealing potential use in virostatic cocktails [11]. Antitumor activity against lymphoma and Ehrlich cell lines was observed for 9 with $\mathrm{LD}_{50}$ in the range of 2.5-3.8 $\mu \mathrm{M}$; antibacterial and antifungal activities were also observed [12]. Compound 10 showed potent activity against the prostate cancer line PC-3 with an IC $_{50}$ of $9.3 \pm 0.2 \mu \mathrm{M}$. Anti-proliferative activity of 9 can be attributed, at least in part, to its ability to induce cellular apoptosis [13]. Compound $\mathbf{1 2}$ exhibited a promising $\mathrm{IC}_{50}>1 \mu \mathrm{g} / \mathrm{mL}$ against three cancer cell lines including murine leukemia (P-388; ATCC: CCL-46), human lung carcinoma (A-549; ATCC: CCL-8) and human colon carcinoma (HT-29; ATCC: HTB-38) [15]. 
Table 1. Aromadendrane sesquiterpenes, sources and activities.

\begin{tabular}{cccc}
\hline No. & Name & Sources & Activities \\
\hline 4 & Guaianediol [10] & Sinularia gardineri & anti-tumor \\
5 & Alismol [11] & Litophyton arboreum & cytostatic \\
6 & Lactiflorenol [17] & Sinularia polydactyla & \\
7 & 10-O-Methyl alismoxide [11] & L. arboreum & \\
8 & Alismoxide [11] & L. arboreum & cytostatic \\
9 & Palustrol [12] & Sarcophyton trocheliophorum & anti-tumor, antibacterial and antifungal \\
10 & 10(14)-Aromadendrene [13] & Sarcophyton glaucum & anti-tumor, antiproliferative \\
11 & Fulfulvene [14] & Parerythropodium & \\
12 & O-Methyl guaianediol [15] & fulvum fulvum & \\
\hline
\end{tabular}

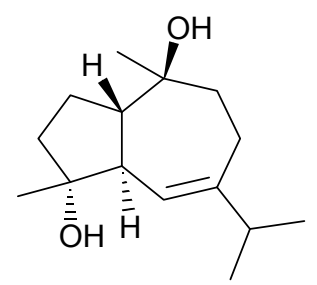

4

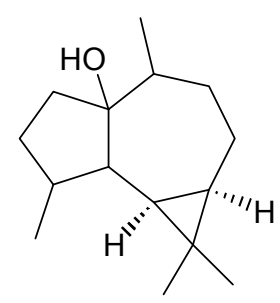

9<smiles>C=C1CCC(C(C)C)=C[C@H]2C(C)CC[C@H]1C2(C)O</smiles>

5<smiles>C=C1CC[C@@H]2[C@H]([C@@H]3C(C)CC[C@@H]13)C2(C)C</smiles>

10

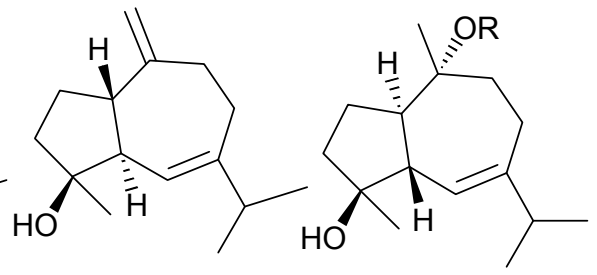

6<smiles>CC1=C2C(=C(C)CCC3C2C3(C)C)C=C1</smiles>

11
$7 \mathrm{R}=\mathrm{Me}$

$8 \mathrm{R}=\mathrm{H}$

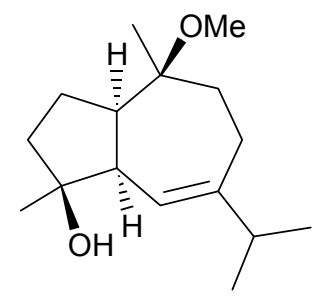

12

Figure 4. Representative structures of aromadendrane-type sesquiterpenes (4-12).

\section{3. $\gamma$-Methoxybutenolide-Type Sesquiterpenes}

Tricyclo-[6,7,5]-sesquiterpenes, Hyrtiosenolide $\mathrm{A}$ and $\mathrm{B}$ have been isolated from the sponge Hyrtios sp., and compounds $\mathbf{1 3}$ and $\mathbf{1 4}$ exhibit weak antibacterial activity against Escherichia coli [16] (Figure 5).

\subsection{Miscellaneous Sesquiterpenes}

Additional sesquiterpenes have been isolated from several coral genera with examples reported in Table 2 and Figure 5. Compound 28 exhibits cytotoxic activity against human hepatocarcinoma (HepG2) and breast adenocarcinoma (MCF-7) [17]. 
Table 2. Other sesquiterpenes, sources and activities.

\begin{tabular}{cccc}
\hline No. & Name & Sources & Activities \\
\hline 15 & 5-Hydroxy-8-methoxy-calamenene [14] & $\begin{array}{c}\text { Parerythropodium } \\
\text { fulvum fulvum } \\
\text { Parerythropodium } \\
\text { fulvum fulvum }\end{array}$ & \\
16 & 5-Hydroxy-8-methoxy-calamenene-6-al [14] & Peyssonnelia sp. & \\
17 & Peyssonol A [17] & Smenospongia sp. & HIV \\
18 & Ilimaquinone [18] & Dysidea cinerea & \\
19 & Avarol [18,19] & D.cinerea & \\
20 & 3'-Hydroxyavarone [20] & D.cinerea & \\
21 & 3',6'-Dihydroxyavarone [20] & D.cinerea & \\
22 & 6'-Acetoxyavarone [20] & D.cinerea & \\
23 & 6'- Hydroxy4'-methoxyavarone [20] & D.cinerea & \\
24 & 6'-Hydroxyavarol [20] & D.cinerea & \\
25 & 6'-Acetoxyavarol [20] & Smenospongia sp. & \\
26 & Smenotronic acid [18] & Smenospongia sp. & \\
27 & Dactyltronic acids [18] & Sarcophyton ehrenbergi & cytotoxic (HepG2) \\
& (E)-Methyl-3-(5-butyl-1-hydroxy-2,3- & & \\
28 & dimethyl-4-oxocyclopent-2-enyl)acrylate [21] & & \\
\hline
\end{tabular}

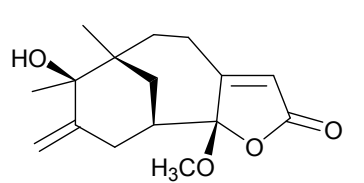

13

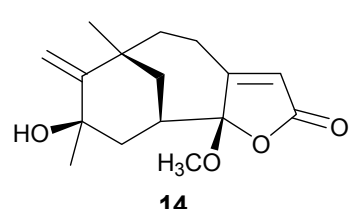

14

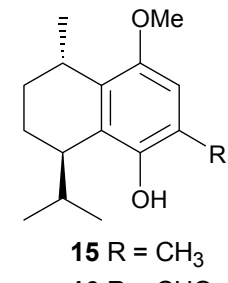

$16 \mathrm{R}=\mathrm{CHO}$

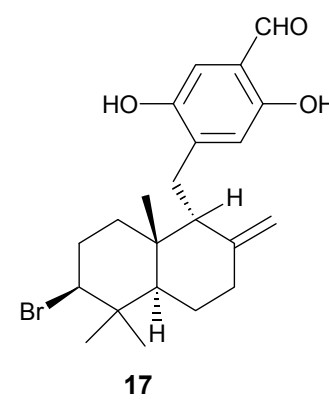

17

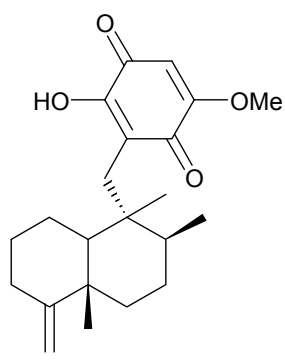

18

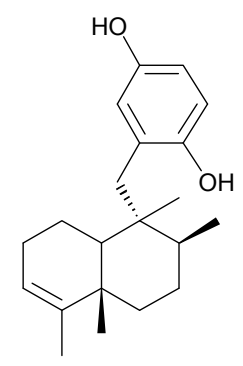

19

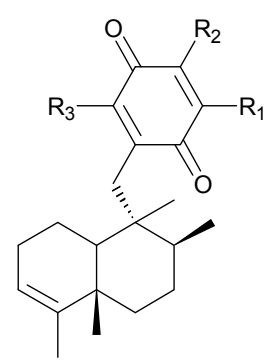

$20 \mathrm{R}_{1}=\mathrm{OH}, \mathrm{R}_{2}=\mathrm{R}_{3}=\mathrm{H}$

$21 \mathrm{R}_{1}=\mathrm{R}_{3}=\mathrm{OH}, \mathrm{R}_{2}=\mathrm{H}$

$22 R_{1}=R_{2}=H, R_{3}=O A C$

$23 \mathrm{R}_{1}=\mathrm{H}, \mathrm{R}_{2}=\mathrm{OCH}_{3}, \mathrm{R}_{3}=\mathrm{OH}$

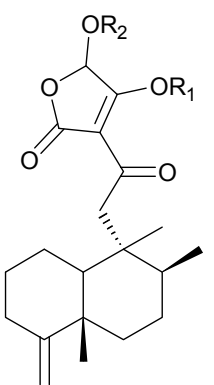

$26 \mathrm{R}_{1}=\mathrm{CH}_{3}, \mathrm{R}_{2}=\mathrm{H}$

$27 \mathrm{R}_{1}=\mathrm{H}, \mathrm{R}_{2}=\mathrm{CH}_{3}$

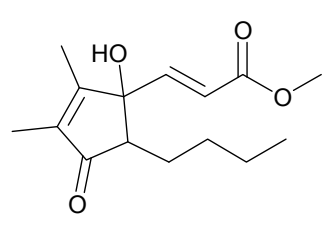

28

$24 \mathrm{R}=\mathrm{H}, \mathrm{R}_{1}=\mathrm{OH}$

$25 \mathrm{R}=\mathrm{H}, \mathrm{R}_{1}=\mathrm{OAC}$

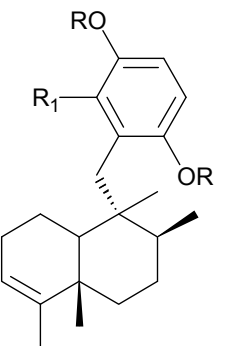

$$
\text { 宊 }
$$

Figure 5. Structures of sesquiterpenes- $\gamma$-methoxybutenolides and sesquiterpene derivatives (13-28).

\section{Diterpenes}

Diterpenoids are widespread in various marine organisms including soft coral (Sarcophyton glaucum, S. trocheliophorum, Sinularia polydactyla, S. gardineri, Litophyton arboreum, Lobophyton sp., Xenia sp. and Cladiella pachyclados) [10-13,22-29], and sponges (Leucetta chagosensis) [23]. 


\subsection{Cembrane-Based Diterpenes}

Fourteen-membered cyclic and bicycle-[5,14]-diterpenes have been isolated from numerous coral genera with examples shown in Table 3 and Figure 6. Compounds 29, 41 and 42 exhibited antibacterial and antifungal activity against Aspergillus flavus and Candida albicans with low $\mu \mathrm{M}$ MIC values [12]. Lack of cytotoxicity against monkey kidney CV-1 cells suggests that 30, 32, and $\mathbf{3 3}$ may prove to be good candidate drugs against melanoma and warrant further studies in the development as antitumor agents [19]. Compound 30 exhibits moderate antifungal activity against Cryptococcus neoformans with an $\mathrm{IC}_{50}$ of $20 \mu \mathrm{g} / \mathrm{mL}$ [22]. Compound 43 showed selective cytotoxicity against HepG2 (IC50 $1.0 \mu \mathrm{g} / \mathrm{mL}$ ) [24]. Compounds 44 and 45 were found to be inhibitors of cytochrome $\mathrm{P}_{450}$ $1 \mathrm{~A}$ activity [25]. Compound 47 exhibits cytotoxic activity against HepG2, HCT-116, and HeLa cells with low $\mathrm{IC}_{50} \mu \mathrm{g} / \mathrm{mL}$ values [26]. Cytotoxic activity against human hepatocarcinoma (HepG2) and breast adenocarcinoma (MCF-7) cell lines was observed for 48 and 49 [21].

Compounds 66 and 68 have significant cytotoxic activity against the human hepatocellular liver carcinoma cell line HepG2 with an $\mathrm{IC}_{50}$ of $20 \mu \mathrm{M}$ while $\mathbf{6 7}$ and $\mathbf{6 8}$ show activity against the human breast adenocarcinoma cell line MCF-7, also with an $\mathrm{IC}_{50}$ in the low $\mu \mathrm{M}$ range. The anti-proliferative activity of 66 and 68 can be attributed, at least in part, to observed cellular apoptosis activity [13,30]. Compound $\mathbf{7 0}$ exhibits cytotoxicity to a variety of cell lines including murine leukemia (P-388), human lung carcinoma (A-549), human colon carcinoma (HT-29) and human melanoma (MEL-28) [31].

Table 3. Cembrane diterpenes, sources and activities.

\begin{tabular}{lccc}
\hline No. & Name & Source & Activity \\
\hline 29 & Cembrene-C [12] & Sarcophyton trocheliophorum & anti-fungal, anti-bacterial \\
& & & anti-tumor, antifungal \\
30 & Sarcophine [19,22] & S. glaucum & \\
31 & $(+)-7 \alpha, 8 \beta$-Dihydroxydeepoxy-sarcophine [22] & S. glaucum & anti-tumor \\
32 & Sarcophytolide 1 [19,30] & S. glaucum & anti-tumor \\
33 & $(1 S, 2 E, 4 R, 7 E, 11 E, 13 S)$-Cembratrien-4,13-diol [22] & S. glaucum & anti-tumor \\
34 & $(1 S, 2 E, 4 R, 6 E, 8 R, 11 S, 12 R)-8,12$-Epoxy-2,6-cembradiene-4,11-diol [22] & S. glaucum & anti-tumor \\
35 & $(1 S, 2 E, 4 R, 6 E, 8 S, 11 R, 12 S)-8,11$-Epoxy-4,12-epoxy-2,6-cembradiene [22] & S. glaucum & \\
36 & Trochelioid A [23] & S. trocheliophorum & \\
37 & Trochelioid B [23] & S. trocheliophorum & \\
38 & 16-Oxosarcophytonin E [23] & S. trocheliophorum & \\
39 & ent-Sarcophine [23] & S. trocheliophorum & S. trocheliophorum \\
\hline
\end{tabular}


Table 3. Cont.

\begin{tabular}{|c|c|c|c|}
\hline 41 & Sarcotrocheliol acetate [12] & S. trocheliophorum & anti-tumor \\
\hline 42 & Sarcotrocheliol [12] & S. trocheliophorum & anti-tumor \\
\hline 43 & Durumolide C [24] & Sinularia polydactyla & anti-fungal, anti-bacterial \\
\hline 44 & 11(S)-Hydroperoxylsarcoph-12(20)-ene [22] & S. glaucum & anti-fungal, anti-bacterial \\
\hline 45 & 12(S)-Hydroperoxylsarcoph-10-ene [25] & S. glaucum & cytotoxic HepG2 (anti-tumor) \\
\hline 46 & $(2 R, 7 R, 8 R)$-Dihydroxy-deepoxysarcophine $[26]$ & S. glaucum & anti-tumor \\
\hline 47 & $7 \beta$-Acetoxy-8 $\alpha$-hydroxy-deepoxysarcophine [26] & S. glaucum & cytotoxic (HepG2)( anti-tumor) \\
\hline 48 & 7-Keto-8 $\alpha$-hydroxy-deepoxysarcophine [21] & S. ehrenbergi & cytotoxic (HepG2) (anti-tumor) \\
\hline 49 & $7 \beta$-Chloro-8 $\alpha$-hydroxy-12-acetoxy-deepoxysarcophine [21] & S. ehrenbergi & cytotoxic (HepG2) (anti-tumor) \\
\hline 50 & Nephthenol [27] & Lobophytum pauciflorum & \\
\hline 51 & Cembrene-A [27] & Alcyonium utinomii & \\
\hline 52 & Alcyonol A [27] & A. utinomii & \\
\hline 53 & Alcyonol B [27] & A. utinomii & \\
\hline 54 & Alcyonol C [27] & A. utinomii & \\
\hline 55 & Pauciflorol A [27] & L. pauciflorum & \\
\hline 56 & Pauciflorol B [27] & L. pauciflorum & \\
\hline 57 & Thunbergol [27] & L. pauciflorum & \\
\hline 58 & Labolide [27] & L. crassum & \\
\hline 59 & 20-Acetylsinularolide B [27] & L. crassum & \\
\hline 60 & 20-Acetylsinularolide C [27] & L. crassum & \\
\hline 61 & Sinularolide C [27] & L. crassum & \\
\hline 62 & Sinularolide C diacetate [27] & L. crassum & \\
\hline 63 & 3-Deoxypresinularolide B [27] & L. crassum & \\
\hline 64 & 3-Deoxy-20-acetylpresinularolide B [27] & L. crassum & \\
\hline 65 & Sarcophytol M [11] & Litophyton arboreum & \\
\hline 66 & Sarcophytolol [13] & Sarcophyton glaucum & $\begin{array}{c}\text { cytotoxic HepG2 (anti-tumor) } \\
\text { antiproliferative }\end{array}$ \\
\hline 67 & Sarcophytolide B [13] & S. glaucum & \\
\hline 68 & Sarcophytolide C [13] & S. glaucum & \\
\hline 69 & Deoxosarcophine [13] & S. glaucum & cytotoxic against MCF-7 (anti-tumor) \\
\hline 70 & 2-epi-Sarcophine [31] & S. auritum & cytotoxic \\
\hline 71 & $(1 R, 2 E, 4 S, 6 E, 8 R, 11 R, 12 R)$-2,6-cembradiene-4,8,11,12-tetrol [31] & S. auritum & cytotoxic \\
\hline 72 & Singardin [31] & Sinularia gardineri & anti-tumor \\
\hline
\end{tabular}




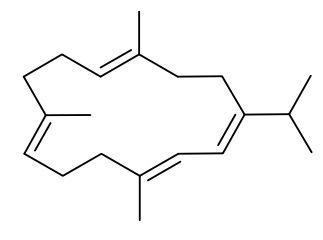

29

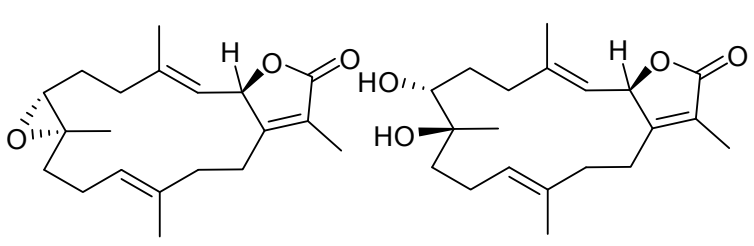

31

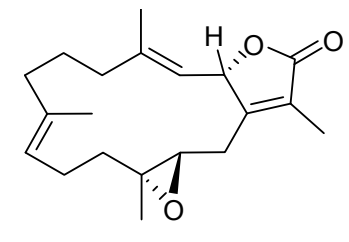

32

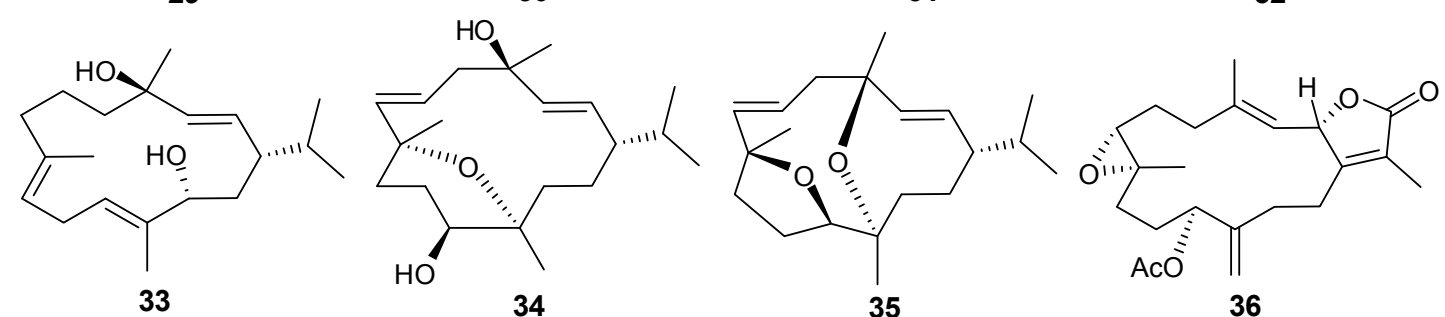

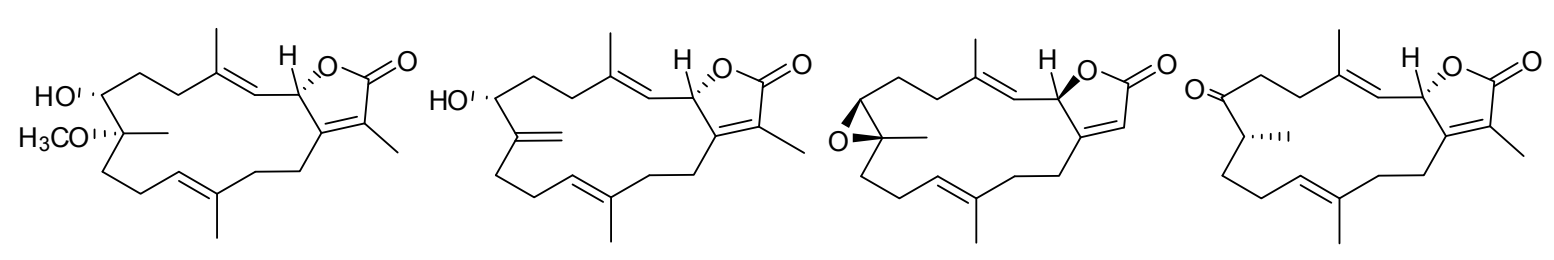

38

39

40<smiles></smiles>

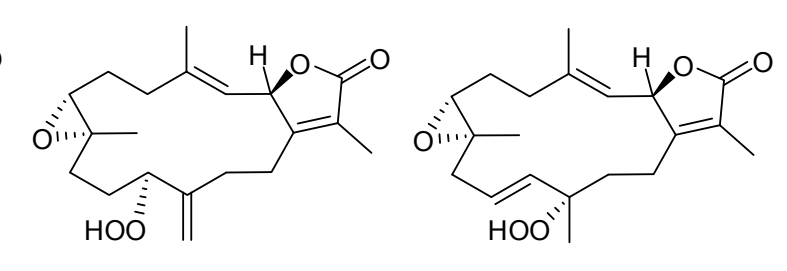

45

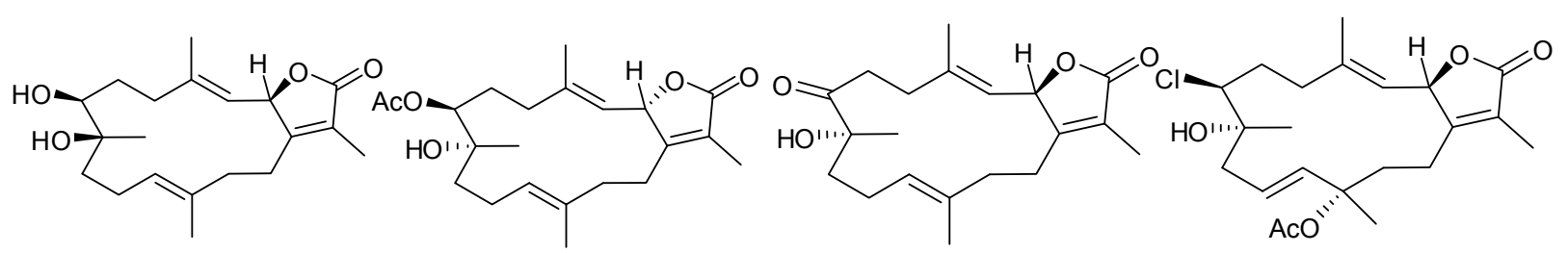<smiles>CC(=CCCC(C)=CCCC(C)(C)O)CCC=C(C)C(C)(C)O</smiles>

50<smiles></smiles>

51

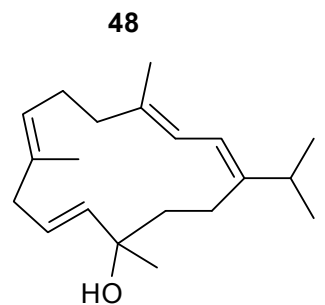

52

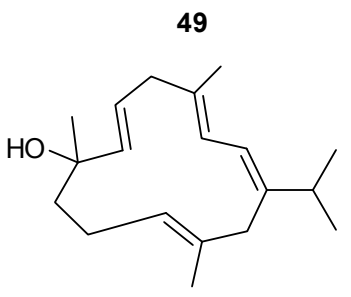

53

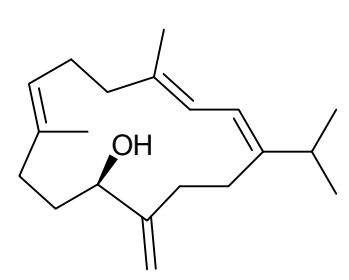

54

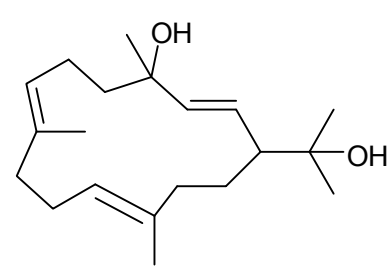

55

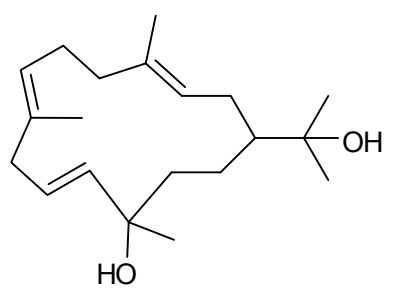

56

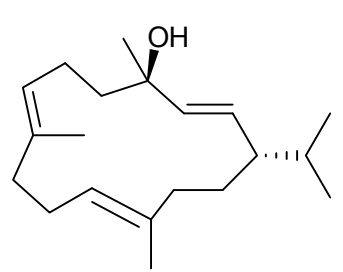

57

Figure 6. Cont. 
<smiles></smiles>

58<smiles>C/C1=C/CC/C(C)=C/C2OC(C)(CCCC1)C(O)C2C(C)C</smiles>

66

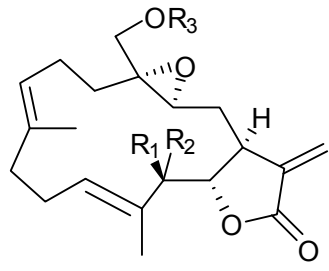

$59 \quad \mathrm{R}_{1}=\mathrm{H}, \quad \mathrm{R}_{2}=\mathrm{OH}, \mathrm{R}_{3}=\mathrm{Ac}$

$60 R_{1}=\mathrm{OH}, \quad \mathrm{R}_{2}=\mathrm{H}, \quad \mathrm{R}_{3}=\mathrm{Ac}$

$61 \mathrm{R}_{1}=\mathrm{OH}, \quad \mathrm{R}_{2}=\mathrm{H}, \quad \mathrm{R}_{3}=\mathrm{H}$

$62 \mathrm{R}_{1}=\mathrm{OAc}, \mathrm{R}_{2}=\mathrm{H}, \quad \mathrm{R}_{3}=\mathrm{Ac}$

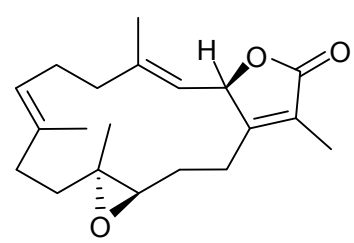

67<smiles>[R6]CC(=CC[C@H]1C(=C)C(=O)O[C@@H]1C/C(C)=C/CC/C(C)=C/CCC)CO</smiles>

$63 \mathrm{R}=\mathrm{H}$

$64 \mathrm{R}=\mathrm{Ac}$<smiles></smiles>

68

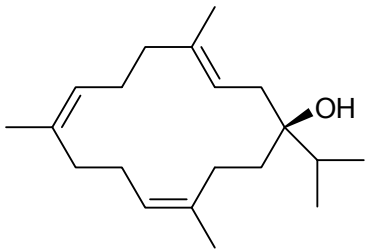

65

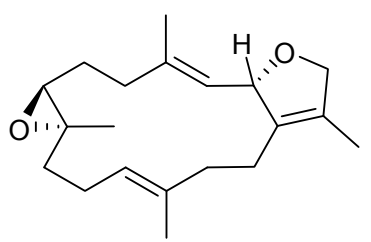

69<smiles></smiles>

70<smiles>CC(C)[C@H]1/C=C/[C@@](C)(O)C/C=C\[C@]2(O)CC[C@](O)(C1)[C@](C)(O)C2</smiles>

71

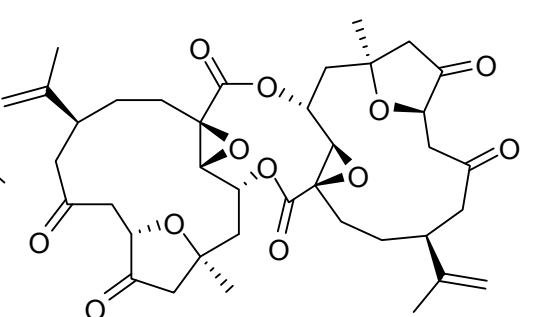

72

Figure 6. Structures of cembrane-based diterpenes (29-72).

\subsection{Xenicane Diterpenes}

Bicyclo-[6,9]/[4,9]-diterpenes have been isolated from the coral genus Xenia with examples shown in Table 4 and Figure 7.

Table 4. Xenia diterpenes, sources and activities.

\begin{tabular}{ccc}
\hline No. & Name & Source \\
\hline 73 & Xenicin [28] & Xenia macrosoiculata \\
74 & Xenialactol-D [28] & X. obscuronata \\
75 & Xenialactol-C [28] & X. obscuronata \\
76 & Xeniolide-E [28] & X. obscuronata \\
77 & 14(15)-Epoxyxeniaphyllene [28] & X. lilielae \\
78 & Xeniaphyllene-dioxide [28] & X. lilielae \\
79 & Xeniaphyllenol-C [28] & X. macrosoiculata \\
80 & Epoxyxeniaphyllenol-A [28] & X. lilielae, X. macrosoiculata \\
81 & 14,15-Xeniaphyllandiol-4,5-epoxide [28] & X. macrosoiculata \\
82 & Xeniaphyllenol-B [28] & X. macrosoiculata \\
\hline
\end{tabular}




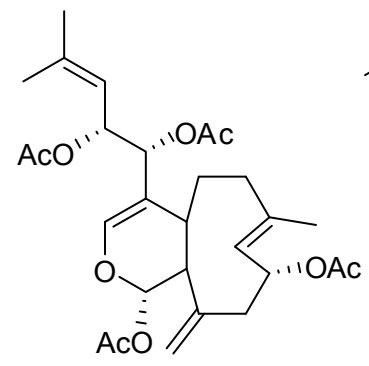

73

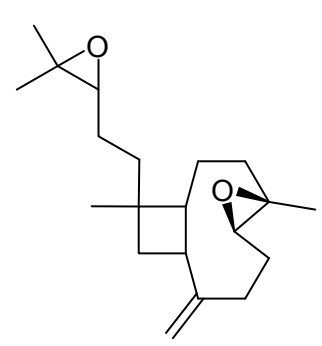

78

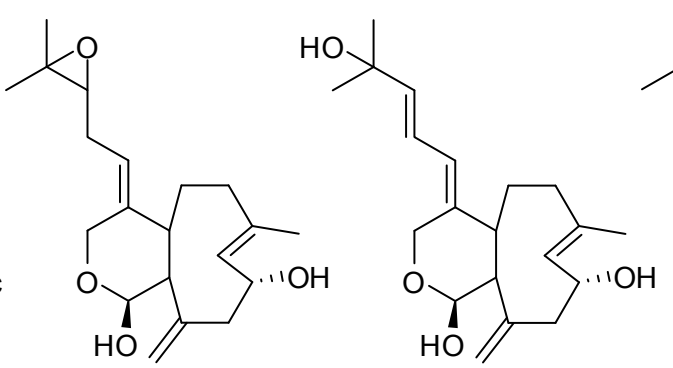

74

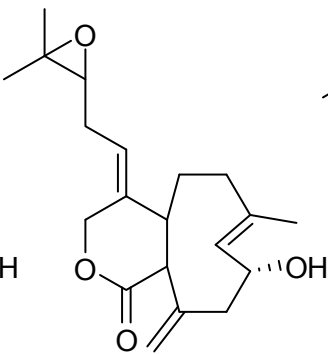

76

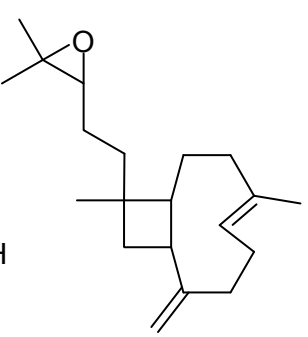

77

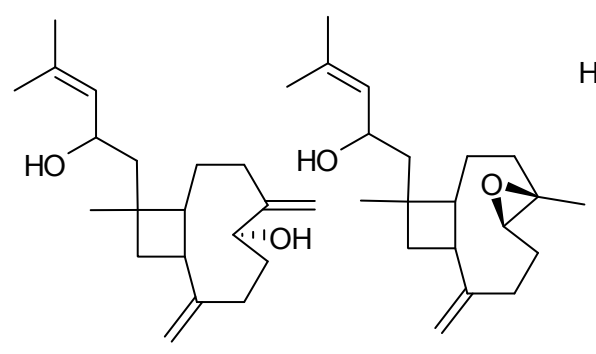

79

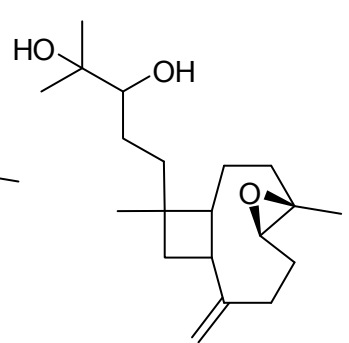

81

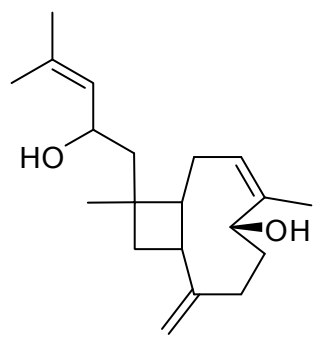

82

Figure 7. The structures of Xenicane diterpenes (73-82).

\subsection{Eunicellin-Based Diterpenes}

Tricyclo-[6,5,10]-diterpenes have been isolated from the soft coral genus Cladiella with examples shown in Table 5 and Figure 8. Eunicellin-based diterpenes display a wide range of bioactivities including anti-inflammatory and antitumor activities [26]. Compounds $\mathbf{8 3 - 1 0 4}$ have been evaluated for activity to inhibit growth, proliferation, invasion and migration of a prostate cancer cell line with potent anti-migratory and anti-invasive activities observed. Compounds with exomethylene functionalities at C-7 and C-11 demonstrate low anti-migratory activity, however replacement of the exomethylene moiety at C-7 with a quaternary oxygenated carbon, appreciatively increases the activity, as observed for compounds 93-94 and 96 [29].

\subsection{Miscellaneous Diterpenes}

Miscellaneous diterpenes were isolated from three different genus Xenia, Chelonaplysilla and Dysidea. These compounds were classified as: prenylated germacrenes (105), bicyclic diterpenes (108, 109), clerodane diterpenes (107), carbo-tricyclic diterpenes (108) and re-arranged spongian diterpenes (110-113) as shown in Table 6 and Figure 9. 
Table 5. Eunicellin diterpenoids, sources and activities.

\begin{tabular}{cccl}
\hline No. & Name & Source & Activity \\
\hline 83 & Pachycladin A [29] & Cladiella pachyclados & anti-tumor, anti-invasive \\
84 & Klysimplexin G [29] & C.pachyclados & anti-tumor, anti-invasive \\
85 & Pachycladin B [29] & C.pachyclados & anti-tumor, anti-invasive \\
86 & Klysimplexin E [29] & C.pachyclados & anti-tumor, anti-invasive \\
87 & Pachycladin C [29] & C.pachyclados & anti-tumor, anti-invasive \\
88 & Cladiellisin [29] & C. pachyclados & anti-tumor, anti-invasive \\
89 & 3-Acetyl cladiellisin [29] & C.pachyclados & anti-tumor, anti-invasive \\
90 & 3,6-Diacetyl cladiellisin [29] & C.pachyclados & anti-tumor, anti-invasive \\
91 & Pachycladin D [29] & C.pachyclados & anti-tumor, anti-invasive \\
92 & Pachycladin E [26] & C.pachyclados & anti-tumor, anti-invasive \\
93 & Sclerophytin A [29] & C.pachyclados & anti-tumor, anti-invasive \\
94 & Sclerophytin F methyl ether [29] & C.pachyclados & anti-tumor, anti-invasive \\
95 & Sclerophytin B [29] & C.pachyclados & anti-tumor, anti-invasive \\
96 & (+)-Polyanthelin A [29] & C. pachyclados & anti-tumor, anti-invasive \\
97 & Cladiella-6Z,11(17)-dien-3-ol [29] & C.pachyclados & anti-tumor, anti-invasive \\
98 & Briarein A [32] & Junceella juncea & \\
99 & Juncins A [32] & J. juncea & \\
100 & Juncins B [32] & J.juncea & \\
101 & Juncins C [32] & J.juncea & \\
102 & Juncins D [32] & J. juncea & \\
103 & Juncins E [32] & J. juncea & \\
104 & Juncins [32] & J.juncea & \\
\hline
\end{tabular}

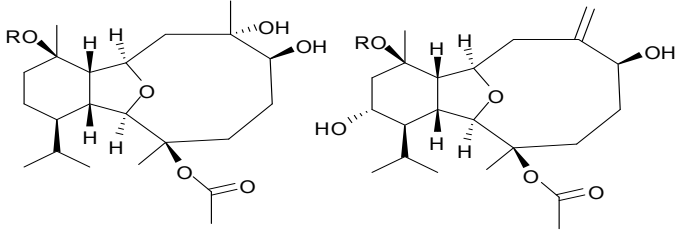
$83 \mathrm{R}=\mathrm{COC}_{3} \mathrm{H}_{7}$
$84 \mathrm{R}=\mathrm{COCH}_{3}$

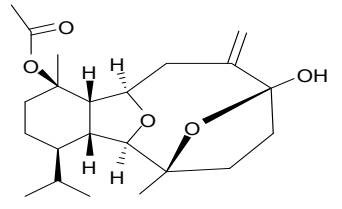

92

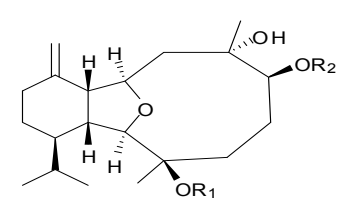

$\begin{array}{lll} & \stackrel{\mathrm{R}_{1}}{\mathrm{R}_{2}} & \mathrm{R}_{2} \\ \mathbf{9 4} & \mathrm{H} & \mathrm{H} \\ \mathbf{9 5} & \stackrel{\mathrm{COCH}}{\mathrm{C}} & \stackrel{\mathrm{H}}{\mathrm{H}}\end{array}$

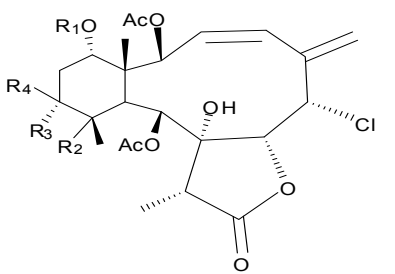

$98 R_{1}=A c, R_{2}=R_{3}=O A c, R_{4}=H$

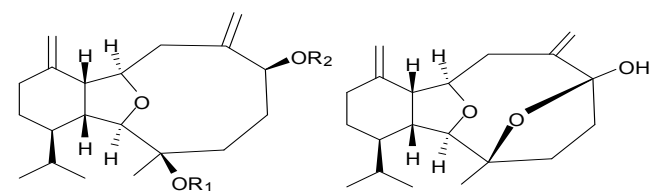

$\begin{array}{lll} & \mathrm{R}_{1} & \mathrm{R}_{2} \\ \mathbf{8 7} & \mathrm{H} & \mathrm{COCH} \\ \mathbf{8 8} & \mathrm{H} & \mathrm{H} \\ \mathbf{8 9} & \mathrm{COCH}_{3} & \mathrm{H} \\ \mathbf{9 0} & \mathrm{COCH}_{3} & \mathrm{COCH}_{3}\end{array}$

91

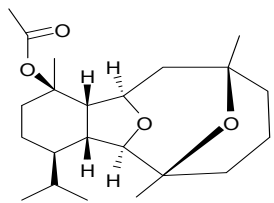

96

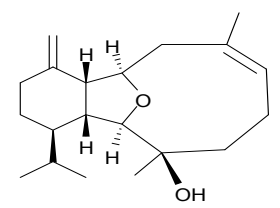

97

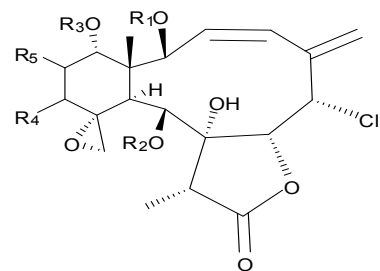

$99 R_{1}=R_{2}=R_{3}=A c, R_{4}=R_{5}=H$

$100 R_{1}=R_{2}=R_{3}=A c, R_{4}=R_{5}=H, 11,20$-deoxy

101 $R_{2}=R_{2}, R_{1}, R_{3}, R_{4}=2 R_{c}=H_{1}, 11,20-d e v a l$.

$103 R_{1}=R_{2}=R_{3}=A c, R_{4}=R_{5}$, $R_{5}=H$

1043,4 dihydro, $R_{1}, R_{2}, R_{3}, O R_{4}=2 A c+$ isobutyrate, $R_{5}=H$

Figure 8. The structure of eunicellin-type diterpenes $(\mathbf{8 3}-\mathbf{1 0 4})$. 
Table 6. Macrocyclic diterpenes, sources and activities.

\begin{tabular}{lcc}
\hline No. & Name & Source \\
\hline 105 & Obscuronatin [28] & Xenia obscuronata \\
106 & Biflora-4,10(19),15-triene [28,33] & X. obscuronata \\
107 & Chelodane [34] & Chelonaplysilla erecta \\
108 & Barekoxide [34] & C. erecta \\
109 & Zaatirin [34] & C. erecta \\
110 & Norrisolide [35] & Dysidea $\mathrm{sp}$. \\
111 & Norrlandin [35] & Dysidea $\mathrm{sp}$. \\
112 & Seco-norrlandin B [35] & Dysidea $\mathrm{sp}$. \\
113 & Seco-norrlandin C [35] & Dysidea $\mathrm{sp}$. \\
\hline
\end{tabular}<smiles>C=CC(C)(O)CC[C@]1(C)C2CCCC(=C)[C@@]2(C)CC[C@@H]1C</smiles><smiles>C=C[C@]1(C)CCC[C@@](C)(CCC2C(=C)CCCC2(C)C)O1</smiles><smiles>C=C([C@H]1COC(=O)[C@H]1CC(C)=O)[C@H]1CCC2C(C)(C)CCC[C@@]21C</smiles><smiles>C=C(COC(C)=O)[C@H]1COC(=O)[C@H]1C[C@H]1CCC2C(C)(C)CCC[C@@]21C</smiles>

Figure 9. The structure of the macrocyclic type diterpenes (105-113).

\section{Sesterterpenes and Norsesterterpenes}

\subsection{Sesterterpenes}

Pentacyclo-[6,6,6,6,5]-sesterterpenes have been isolated from two different sponges with examples shown in Table 7 and Figure 10. Compound 116 exhibits antimycobacterial inhibition against Mycobacterium tuberculosis (H37Rv) at a concentration of $6 \mu \mathrm{g} / \mathrm{mL}$ while 117-119 displayed moderate to weak inhibitory activity [36]. Compounds 122-123 showed significant cytotoxicity against murine leukemia (P-388), human lung carcinoma (A-549) and a human colon carcinoma (HT-29) [37]. 
Table 7. Sesterterpenes, sources and activities.

\begin{tabular}{lccl}
\hline No. & Name & Source & Activity \\
\hline 114 & Scalardysin [18] & Dysidea herbacea & \\
115 & 25-Dehydroxy-12-epi-deacetylscalarin [36] & Hyrtios erecta & antimycobacterial \\
116 & Sesterstatin [36] & H. erecta & antimycobacterial \\
117 & 16-epi-Scalarolbutenolide [36] & H. erecta & antimycobacterial \\
118 & 3-Acetylsesterstatin [36] & H. erecta & antimycobacterial \\
119 & Salmahyrtisol A [37] & H. erecta & \\
120 & Hyrtiosal [37] & H. erecta & \\
121 & Salmahyrtisol B [37] & H. erecta & cytotoxic (anti-tumor) \\
122 & 19-Acetyl sesterstatin [37] & H. erecta & cytotoxic (anti-tumor) \\
123 & Scalarolide [37] & H. erecta & \\
124 & Salmahyrtisol C [37] & H. erecta & \\
125 & 16-Hydroxyscalarolide [38] & H. erecta & Cytotoxic, antimycobacterial \\
126 & 12-O-Deacetyl-12-epi-scalarine [38] & H. erecta & Cytotoxic, antimycobacterial \\
127 & (-)-Wistarin [39] & Ircinia wistarii & \\
128 & (+)-Wistarin [39] & I. wistarii & \\
129 & $(-)-$ Ircinianin [39] & I. wistarii & \\
130 & Bilosespens A [40] & Dysidea cinerea & Cytotoxic \\
131 & Bilosespens A [40] & D. cinerea & Cytotoxic \\
\hline
\end{tabular}

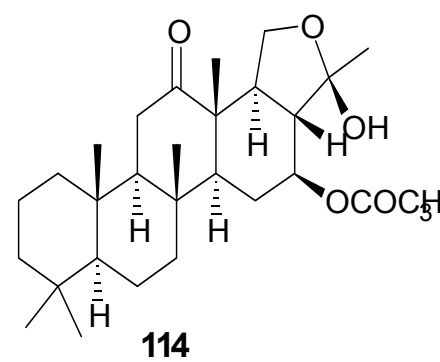

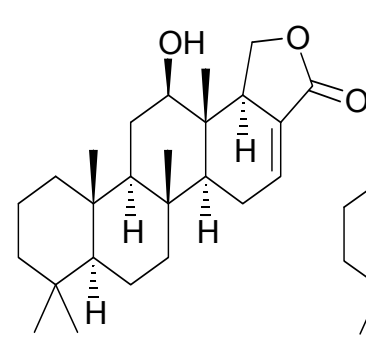

115

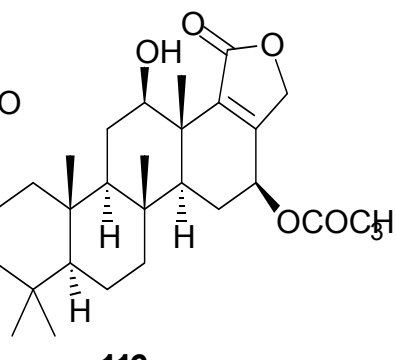

116

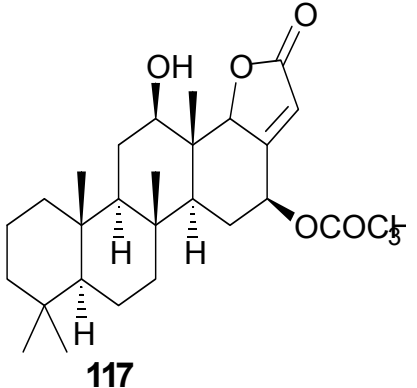

117

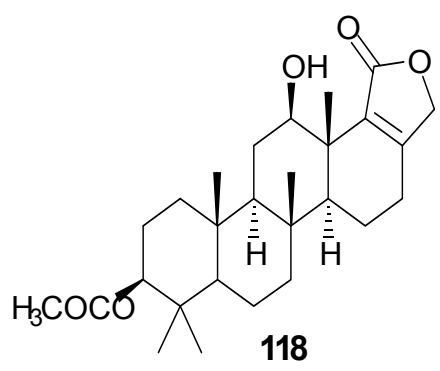<smiles>CC(=O)O[C@H]1c2occc2[C@H](O)C[C@H]2[C@H]3CCC4C(C)(C)CCC[C@]4(C)[C@H]3C[C@@]21C</smiles>

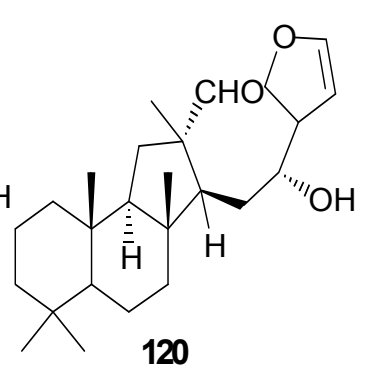

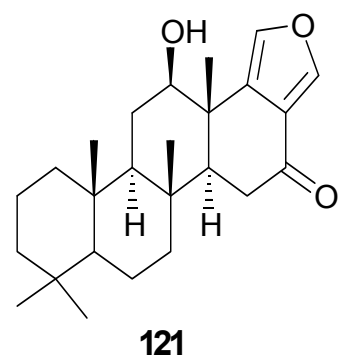

Figure 10. Cont. 


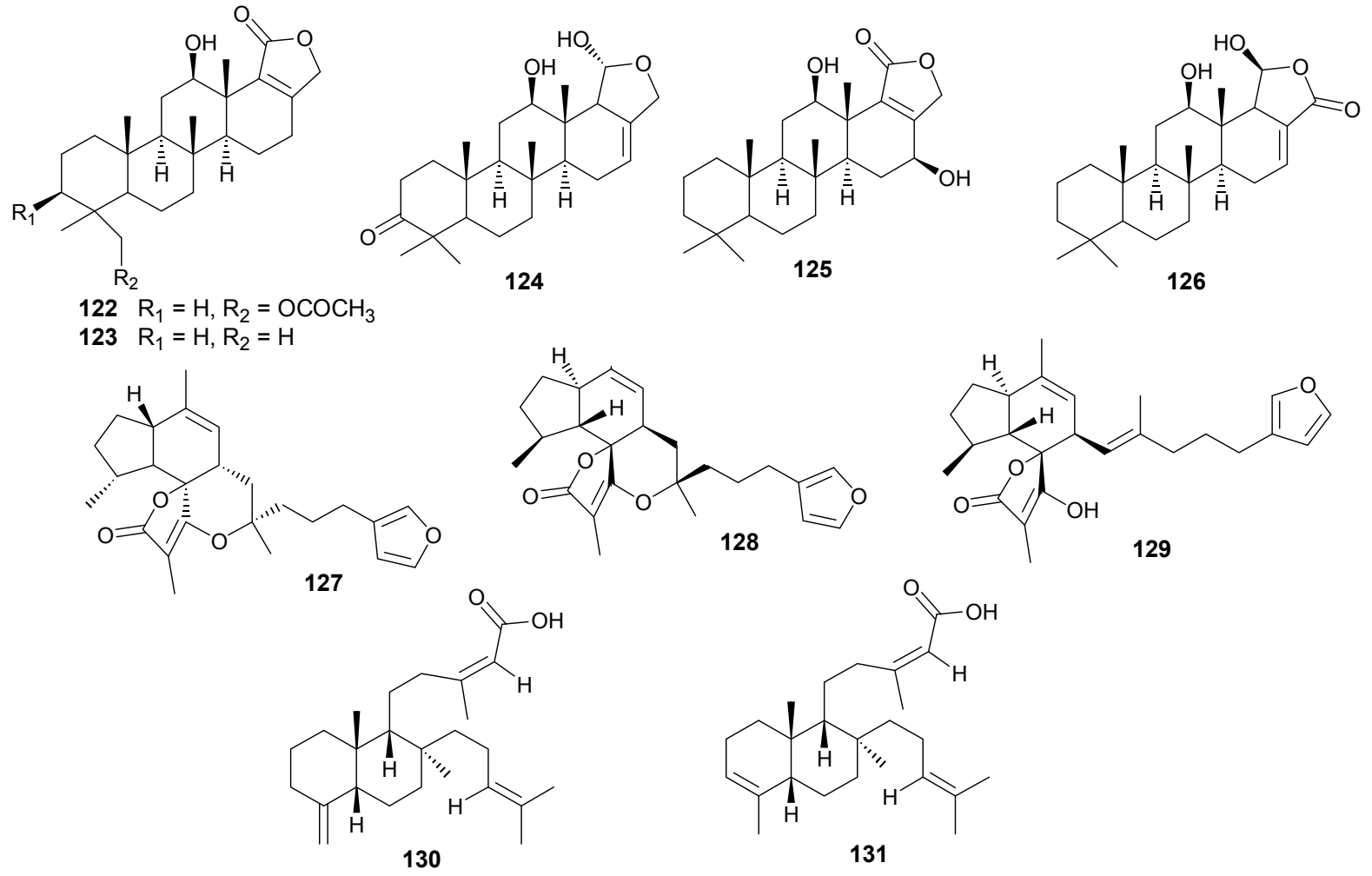

Figure 10. Structures of sesterterpenes (114-131).

\subsection{Norsesterterpenes}

Norsesterterpenes have been isolated from the sponge species Diacarnus erythraeanus with examples shown in Table 8 and Figure 11. Antitumor natural peroxide products are known to induce cytotoxicity in cancer cells through the generation of particular reactive oxygen species (ROSs). Compounds 134-135 displayed mean $\mathrm{IC}_{50}$ growth inhibitions less than $10 \mu \mathrm{M}$ with several tumor cell lines [41]. However, additional studies with 135 established no in vitro selective growth inhibition between normal and tumor cells. In assaying three cancer cells including murine leukemia (P-388), human lung carcinoma (A-549) and human colon carcinoma (HT-29), 140-143 exhibited an IC50 greater than $1 \mu \mathrm{g} / \mathrm{mL}$ [15] while 145 showed lower cytotoxicity against the same lines [42].

Table 8. Norsesterterpenes, sources and activities.

\begin{tabular}{cccc}
\hline No. & Name & Source & Activity \\
\hline 132 & Nuapapuin A methyl ester [41] & Diacarnus erythraeanus & \\
133 & Methyl-2-epinuapapuanoate [41] & D. erythraeanus & \\
134 & $(-)-13,14-$-Epoxymuqubilin A [41] & D. erythraeanus & anti-tumor \\
135 & $(-)-9,10$-Epoxymuqubilin A [41] & D. erythraeanus & anti-tumor \\
136 & $(-)-M u q u b i l i n$ A [41,43] & D. erythraeanus & anti-tumor \\
137 & Hurghaperoxide [41] & D. erythraeanus & \\
138 & Sigmosceptrellin B [41] & D. erythraeanus & \\
139 & Sigmosceptrellin B methyl ester [41] & D. erythraeanus & \\
140 & Aikupikoxide A [15] & D. erythraeanus & cytotoxic \\
\hline
\end{tabular}


Table 8. Cont.

\begin{tabular}{lclc}
\hline 141 & Aikupikoxide D [15] & D. erythraeanus & cytotoxic \\
142 & Aikupikoxide C [15] & D. erythraeanus & cytotoxic \\
143 & Aikupikoxide B [15] & D. erythraeanus & cytotoxic \\
144 & Tasnemoxide A [42] & D. erythraeanus & cytotoxic (anti-tumor) \\
145 & Tasnemoxide B [42] & D. erythraeanus & cytotoxic (anti-tumor) \\
146 & Tasnemoxide C [42] & D. erythraeanus & cytotoxic (anti-tumor) \\
147 & epi-Sigmosceptrellin B [44] & D. erythraeanus & \\
148 & Muqubilone [45] & D. erythraeanus & antimalarial \\
\hline
\end{tabular}

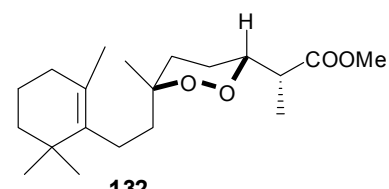<smiles>CC(=O)CC(C)COC(C)CCC1=C(C)CCCC1(C)C</smiles>

133

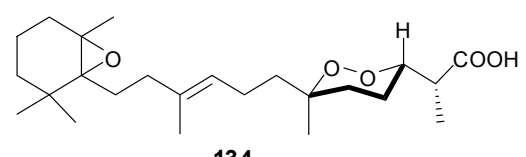

134

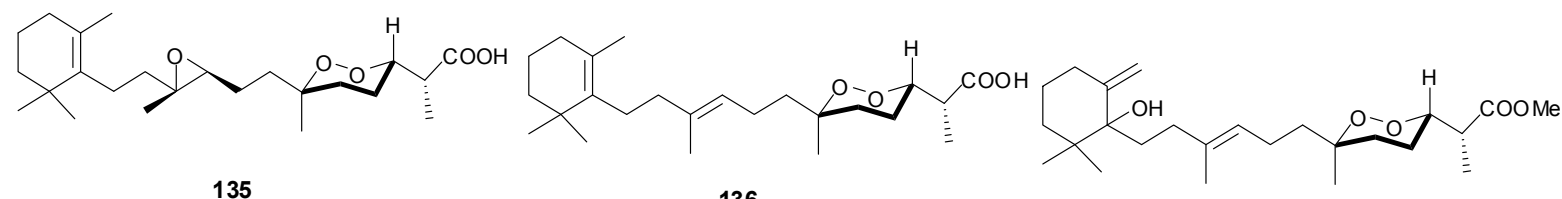

137

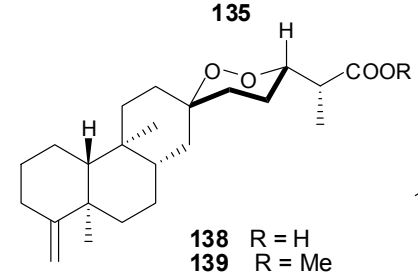

136

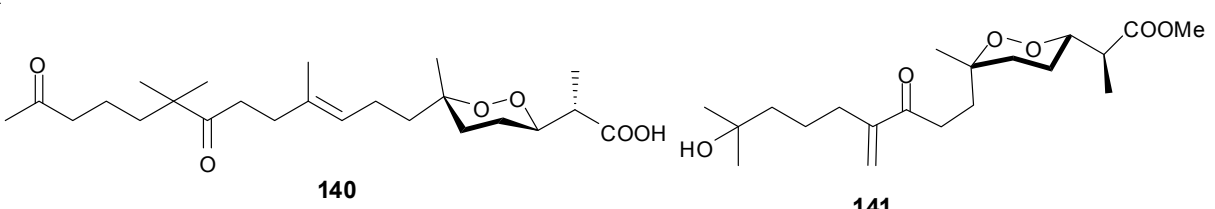

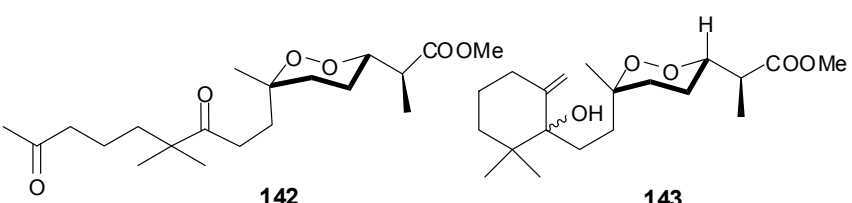<smiles></smiles>

143<smiles>C=C(CCCC1(C)CCC(C(C)OC(C)=O)OO1)CCC1(O)C(=C)CCCC1(C)C</smiles>

144

146

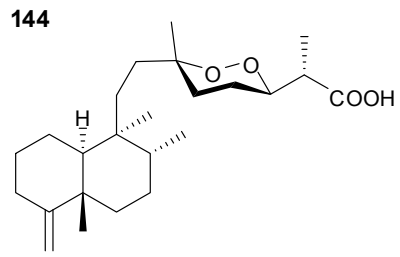

147

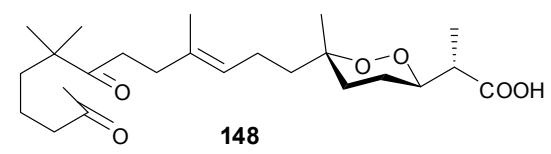

Figure 11. Structures of norterpenes (132-148).

\section{Triterpenes}

Structurally diverse triterpenes are widespread in Red Sea sponges with examples shown in Table 9 and Figure 12. Compound 149 inhibits growth of human breast cancer cells, MDA-MB-231, MCF-7, BT-474 and T-47D, in a dose-dependent manner [46,47]. Triterpenes have also been studied for their efficacy in reducing the appearance of drug resistance. In the presence of many cytotoxic drugs, resistant cell variants appear, a process referred to as multidrug resistance (MDR). Overexpression of 
the ATP-binding cassette (ABC) transporter ABCB1/P-glycoprotein (P-gp) is one of the most common causes of MDR in cancer cells. P-gp a 170-kD transmembrane glycoprotein functions as a drug efflux pump that extrudes a wide spectrum of compounds including amphipathic and hydrophobic drugs. Sipholane triterpenoids can serve as P-gp inhibitors and are being developed to enhance the effect of chemotherapeutic drugs with MDR cancer cells in vitro and in vivo [33,36]. Compounds 162-163 enhanced cytotoxicity of several P-gp substrate-anticancer drugs, including colchicine, vinblastine and paclitaxel. These sipholane triterpenes significantly reversed the MDR-phenotype in P-gp-over expressing MDR cancer cells, KB-C2, in a dose-dependent manner. Moreover, these sipholanes have no effect on the response to cytotoxic agents in cells lacking P-gp expression or expressing MRP1 $(\mathrm{ABCC} 1)$ or MRP7 (ABCC10) or with the breast cancer resistance protein (BCRP/ABCG2). Perhaps most importantly, these sipholanes with a low $\mathrm{IC}_{50}$ of $c a .50 \mu \mathrm{M}$ are not toxic to the assayed cell lines [48].

Table 9. Triterpenes, sources and activities.

\begin{tabular}{|c|c|c|c|}
\hline No. & Name & Source & Activity \\
\hline 149 & Neviotine-A $[46,47]$ & Siphonochalina siphonella & \\
\hline 150 & Sipholenol A [47,49-53] & S. siphonella & anti-tumor \\
\hline 151 & SipholenolA-4-O-3',4'-dichlorobenzoate [49] & S. siphonella & \\
\hline 152 & Shaagrockol B [54] & Toxiclona toxius & \\
\hline 153 & Shaagrockol C [54] & T. toxius & \\
\hline 154 & Sipholenol G [55] & S. siphonella & \\
\hline 155 & Sipholenone D [55] & S. siphonella & \\
\hline 156 & Sipholenol F [55] & S. siphonella & \\
\hline 157 & Sipholenol H [55] & S. siphonella & \\
\hline 158 & Neviotine B [55] & S. siphonella & \\
\hline 159 & Sipholenoside A [55] & S. siphonella & \\
\hline 160 & Sipholenoside B [55] & S. siphonella & \\
\hline 161 & Siphonellinol B [55] & S. siphonella & \\
\hline 162 & Dahabinone A [55] & S. siphonella & \\
\hline 163 & Sipholenone E [51] & S. siphonella & anti-tumor \\
\hline 164 & Sipholenol L [47,51] & S. siphonella & anti-tumor \\
\hline 165 & Sipholenol J [51] & S. siphonella & \\
\hline 166 & $\begin{array}{c}(2 S, 4 \mathrm{a} S, 5 S, 6 R, 8 \mathrm{a} S)-5-(2-((1 S, 3 \mathrm{a} S, 5 R, 8 \mathrm{a} S, Z)- \\
\text { 1-hydroxy-1,4,4,6-tetramethyl-1,2,3,3a,4,5,8,8a-octahydroazulen-5-yl)-ethyl)- } \\
\text { 4a,6-dimethyloctahydro-2H-chromene-2,6-diol [51] }\end{array}$ & S. siphonella & \\
\hline 167 & Sipholenol K [51] & S. siphonella & \\
\hline 168 & Sipholenol M [51] & S. siphonella & \\
\hline 169 & Siphonellinol D [51] & S. siphonella & \\
\hline 170 & Siphonellinol E [51] & S. siphonella & \\
\hline
\end{tabular}


Table 9. Cont.

\begin{tabular}{|c|c|c|c|}
\hline 171 & Siphonellinol-C-23-hydroperoxide [51] & S. siphonella & \\
\hline 172 & Siphonellinol C [56] & S. siphonella & \\
\hline 173 & epi-Sipholenol I [56] & S. siphonella & \\
\hline 174 & Sipholenol I [51] & S. siphonella & \\
\hline 175 & Sipholenone A [56,47] & S. siphonella & \\
\hline 176 & Sipholenol D [52] & S. siphonella & \\
\hline 177 & Neviotine-C [47] & Siphonochalina siphonella & cytotoxic \\
\hline
\end{tabular}

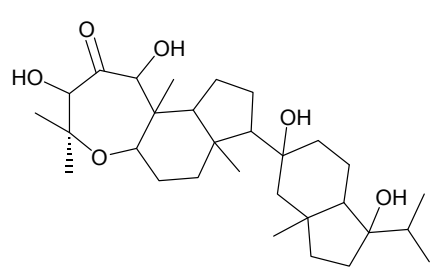

149

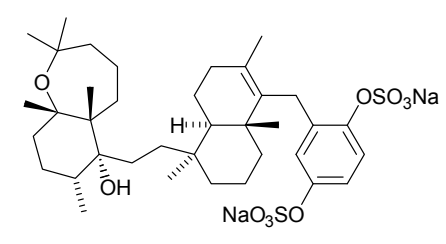

153

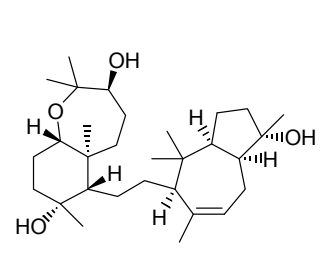

150

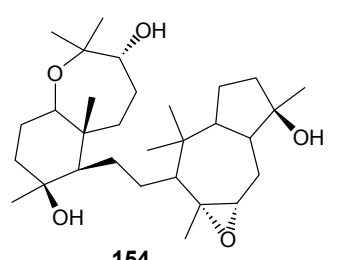

154

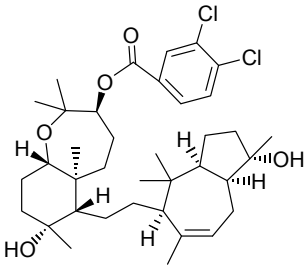

151

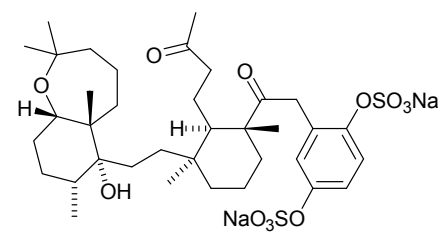

152

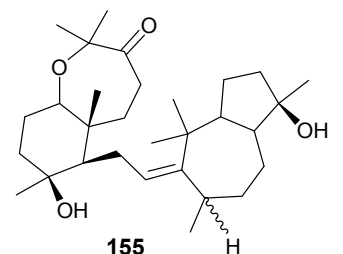<smiles>CC1=CCC2C1CC[C@](C)(O)C(C)(C)C2CCC12CCC(O)C(C)(C)C1CCC2(C)O</smiles>

155
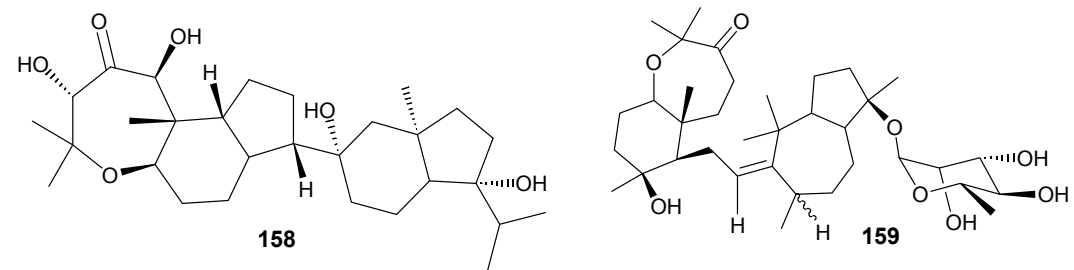

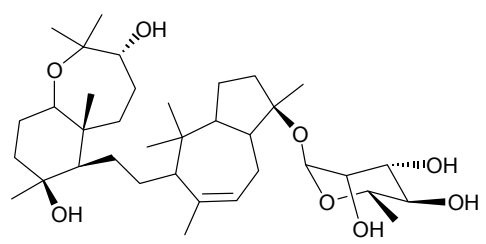

160
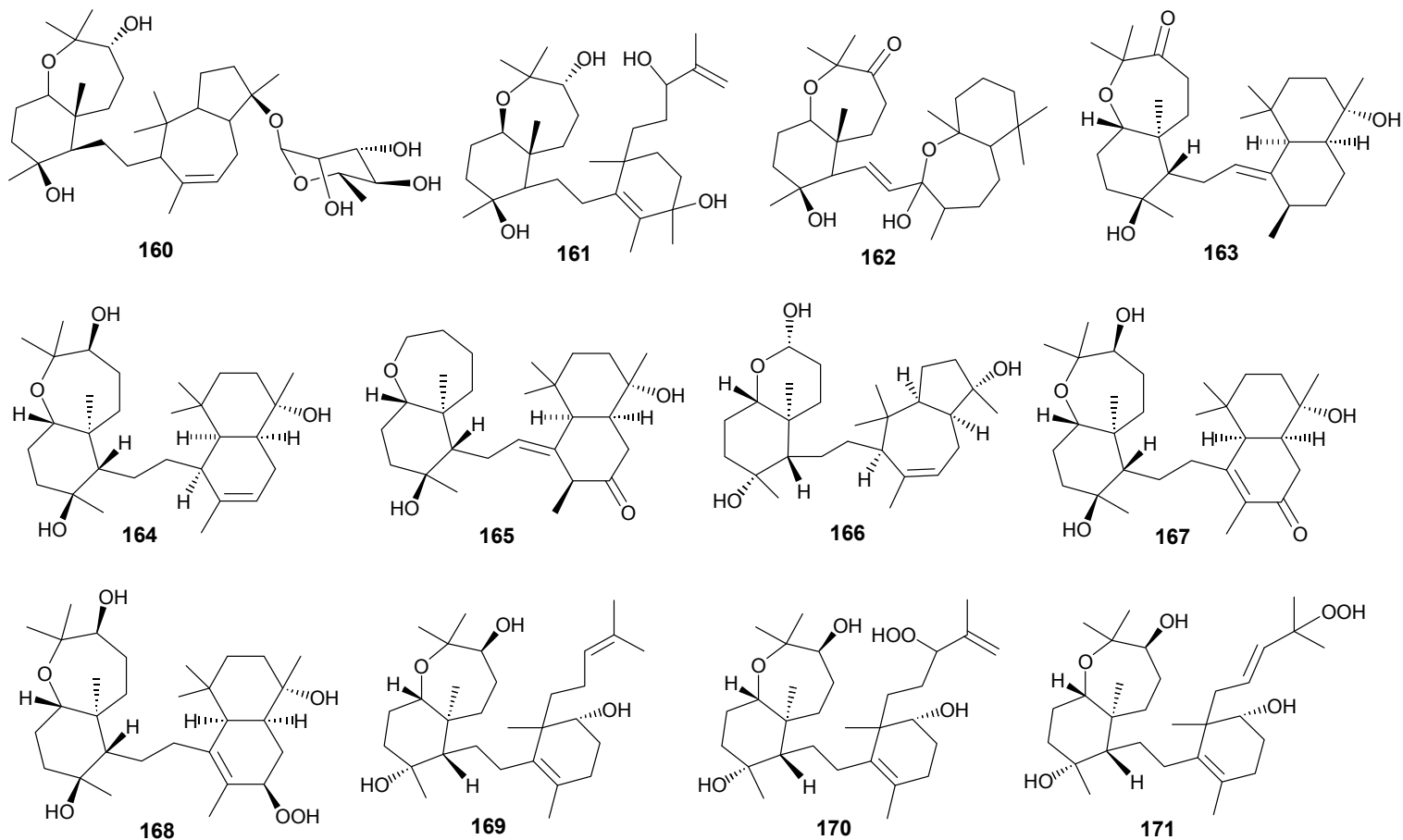

Figure 12. Cont. 

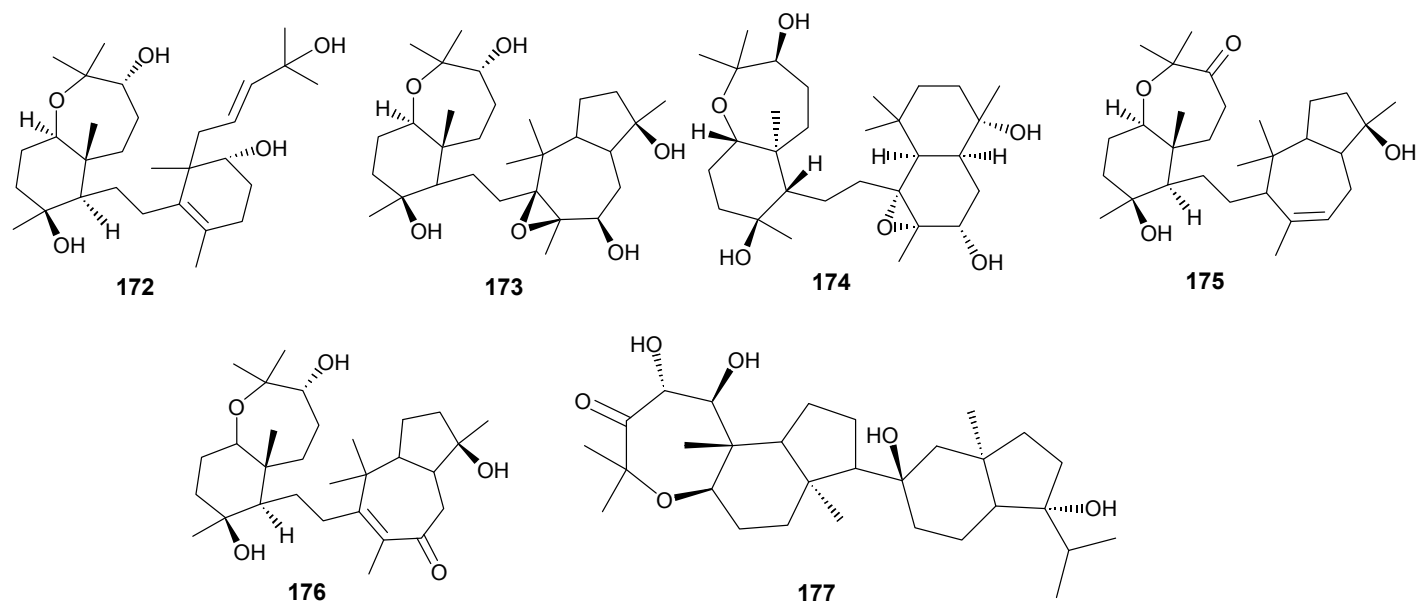

Figure 12. Structures of triterpenes (149-177).

\section{Steroids}

Steriods are widespread throughout the marine biome with recent chemical reports including soft coral (Sinularia candidula, S. polydactyla, Heteroxenia ghardaqensis, Dendronephthya sp., Lobophytom depressum and Litophyton arboreum) [9,11,24,57-60], black coral (Antipathes dichotoma) [38,40], and sponges (Echinoclathria gibbosa, Hyrtios sp., Erylus sp., and Petrosia sp.) [18,64-66]. Steroid examples are shown in Table 10 and Figure 13.

Table 10. Steroids, sources and activities.

\begin{tabular}{|c|c|c|c|}
\hline No. & Name & Source & Activity \\
\hline 178 & 3 $\beta$-25-Dihydroxy-4-methyl-5 $\alpha, 8 \alpha$-epidioxy-2-ketoergost-9-ene [57] & Sinularia candidula & anti-viral \\
\hline 179 & 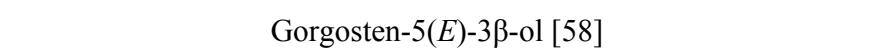 & Heteroxenia ghardaqensis & anti-tumor \\
\hline 180 & Gorgostan-3 $\beta, 5 \alpha, 6 \beta, 11 \alpha$-tetraol (sarcoaldosterol A) [58] & H. ghardaqensis & \\
\hline 181 & Gorgostan-3 $\beta, 5 \alpha, 6 \beta$-triol-11 $\alpha$-acetate $[58]$ & H. ghardaqensis & \\
\hline 182 & 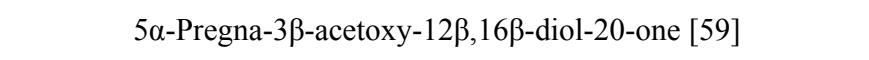 & Echinoclathria gibbosa & anti-tumor \\
\hline 183 & $\beta$-Sitosterol-3-O-(3Z)-pentacosenoate [59] & E. gibbosa & anti-tumor \\
\hline 184 & Cholesterol [9] & Dendronephthya & \\
\hline 185 & Dendronesterone A [9] & Dendronephthya & \\
\hline 186 & 24-Methylcholestane-3 $\beta, 5 \alpha, 6 \beta, 25$-tetrol-25-monoacetate [24] & Sinularia polydactyla & anti-tumor \\
\hline 187 & 24-Methylcholestane-5-en-3ß,25-diol [24] & S. polydactyla & antimicrobial \\
\hline 188 & Lobophytosterol $[60]$ & L. depressum & \\
\hline 189 & 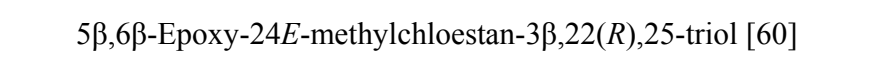 & L. depressum & \\
\hline 190 & Depresosterol $[60]$ & L. depressum & \\
\hline
\end{tabular}


Table 10. Cont.

\begin{tabular}{|c|c|c|c|}
\hline 191 & 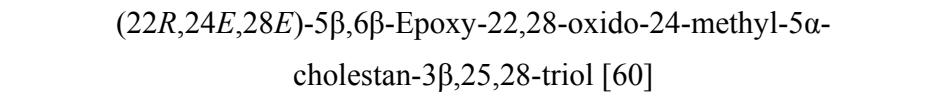 & L. depressum & \\
\hline 192 & 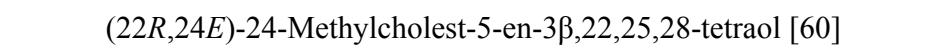 & L. depressum & \\
\hline 193 & 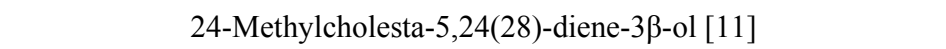 & Litophyton arboreum & \\
\hline 194 & $7 \beta$-Acetoxy-24-methylcholesta-5-24(28)-diene-3,19-diol [11] & L. arboreum & cytotoxic \\
\hline 195 & 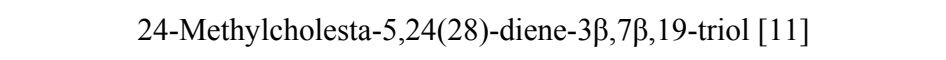 & L. arboreum & \\
\hline 196 & Hyrtiosterol [16] & Hyrtios Species & \\
\hline 197 & Eryloside A [61,62,63] & Genus Erylus & cytotoxic \\
\hline 198 & (22E)-Methylcholesta-5,22-diene-1 $\alpha, 3 \beta, 7 \alpha$-triol [64] & Antipathes dichotoma & anti-bacterial \\
\hline 199 & $3 \beta, 7 \alpha$-Dihydroxy-cholest-5-ene $[64]$ & A. dichotoma & anti-bacterial \\
\hline 200 & 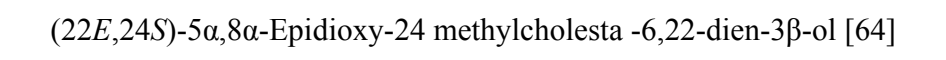 & A. dichotoma & anti-bacterial \\
\hline 201 & $(22 E, 24 S)-5 \alpha, 8 \alpha$-Epidioxy-24-methylcholesta-6,9(11),22-trien-3 $\beta$-ol [64] & A. dichotoma & anti-bacterial \\
\hline 202 & $3 \beta$-Hexadecanoylcholest-5-en-7-one [65] & A. dichotoma & anti-tumor \\
\hline 203 & 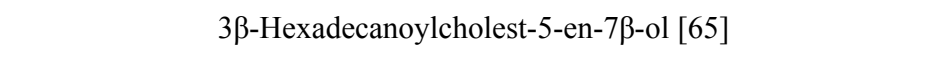 & A. dichotoma & anti-tumor \\
\hline 204 & Cholest-5-en-3 $\beta$-yl-formate [65] & A. dichotoma & anti-tumor \\
\hline 205 & $3 \beta$-Hydroxycholest-5-en-7-one [65] & A. dichotoma & \\
\hline 206 & Cholest-5-en-3 $\beta, 7 \beta$-diol [65] & A. dichotoma & \\
\hline 207 & 22-Dehydrocholestrol [65] & A. dichotoma & \\
\hline 208 & $3 \beta, 7 \beta, 9 \alpha$-Trihydroxycholest-5-en [66] & Petrosia & cytotoxic (anti-tumor) \\
\hline 209 & 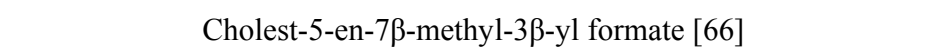 & Petrosia sp. & cytotoxic (anti-tumor) \\
\hline 210 & Dehydroepiandrosterone $[66]$ & Petrosia sp. & cytotoxic (anti-tumor) \\
\hline 211 & 7-Dehydrocholesterol $[66]$ & Petrosia sp. & cytotoxic (anti-tumor) \\
\hline 212 & $5 \alpha, 6 \alpha$-Epoxycholest-8(14)-ene-3 $\beta, 7 \alpha$-diol [66] & Petrosia sp. & cytotoxic (anti-tumor) \\
\hline 213 & 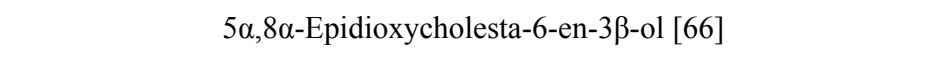 & Petrosia sp. & cytotoxic (anti-tumor) \\
\hline 214 & Cholesta-8-en-3 $\beta, 5 \alpha, 6 \alpha, 25$-tetrol [67] & $\begin{array}{l}\text { Lamellodysidea } \\
\text { herbacea }\end{array}$ & \\
\hline 215 & Cholesta-8(14)-en-3 $\beta, 5 \alpha, 6 \alpha, 25$-tetrol [67] & L. herbacea & \\
\hline 216 & Cholesta-8,24-dien-3 $\beta, 5 \alpha, 6 \alpha$-triol [67] & L. herbacea & anti-fungal \\
\hline 217 & Cholesta-8(14),24-dien-3 $\beta, 5 \alpha, 6 \alpha$-triol [67] & L. herbacea & anti-fungal \\
\hline 218 & Clathsterol [68] & Clathria sp. & \\
\hline 219 & Clionasterol [69] & Dragmacidon coccinea & \\
\hline 220 & Stigmasterol [69] & D. coccinea & \\
\hline 221 & Campesterol [69] & D. coccinea & \\
\hline 222 & Brassicasterol [69] & D. coccinea & \\
\hline 223 & Dendrotriol [70] & $\begin{array}{l}\text { Dendronephthya } \\
\text { hemprichi }\end{array}$ & \\
\hline 224 & Erylosides K [62] & Erylus lendenfeldi & \\
\hline 225 & Erylosides L [62] & E. lendenfeldi & \\
\hline 226 & Erylosides B [63] & E. lendenfeldi & \\
\hline
\end{tabular}




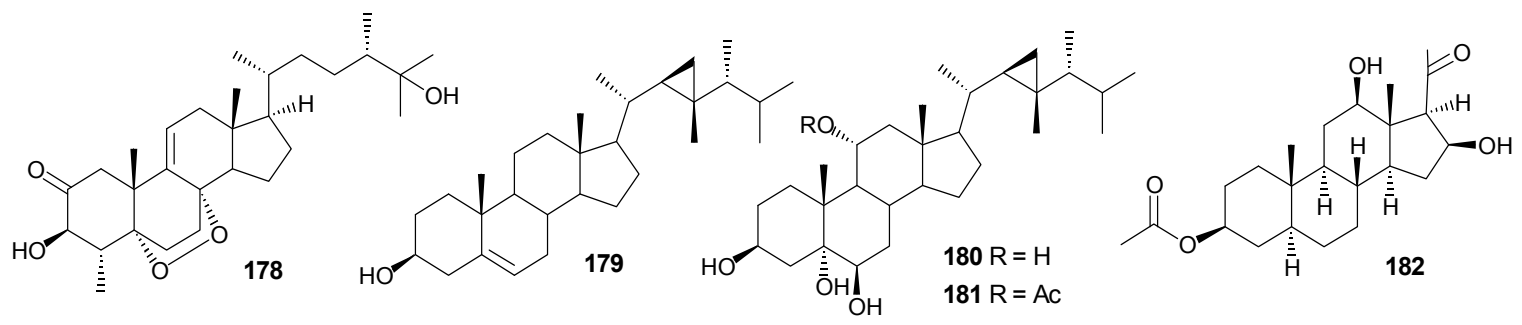

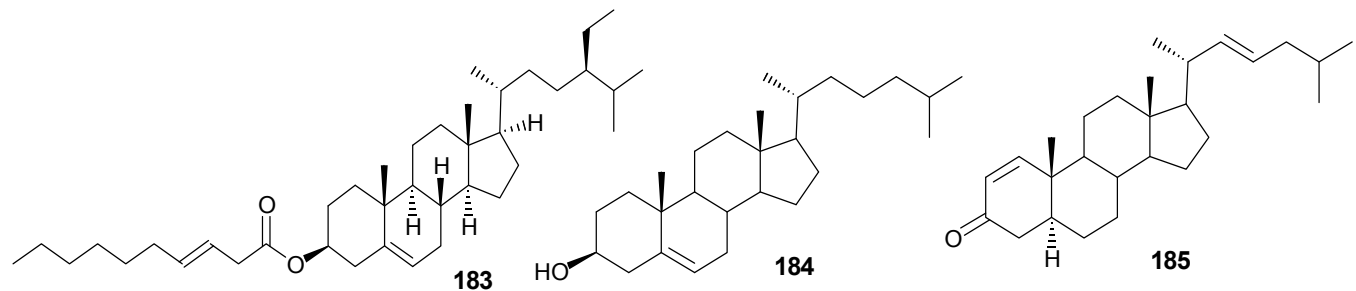
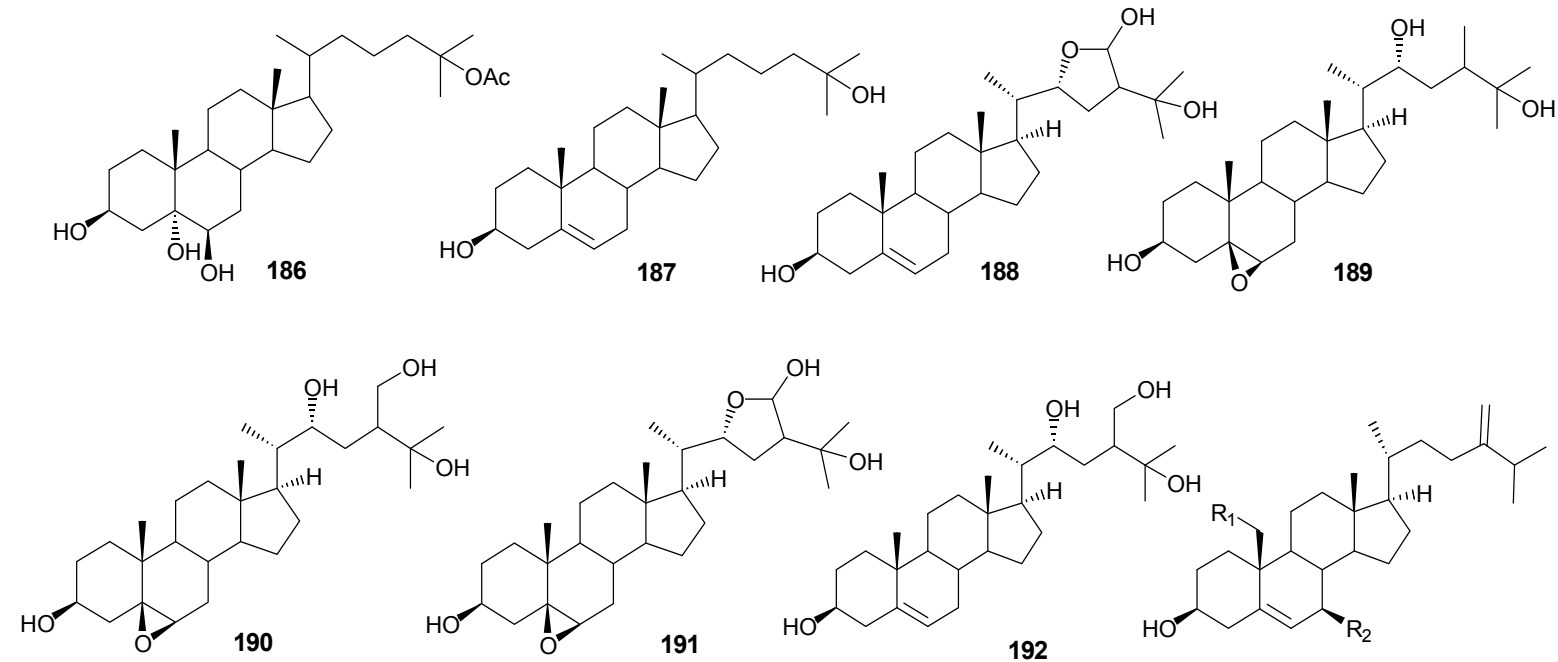

$193 \mathrm{R}_{1}=\mathrm{R}_{2}=\mathrm{H}$

$194 \mathrm{R}_{1}=\mathrm{OH} \quad \mathrm{R}_{2}=\mathrm{OAc}$

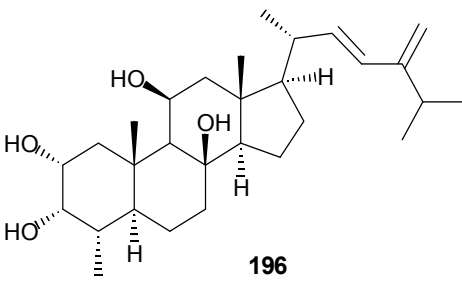

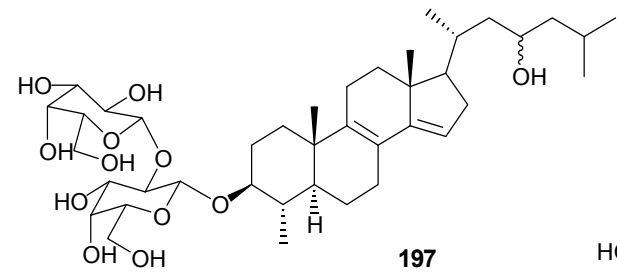<smiles>CC(C)CC(C)/C=C/C(C)[C@H]1CCC2C3C(O)C=C4CC(O)C[C@H](O)C4(C)C3CC[C@]21C</smiles>

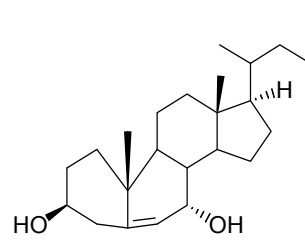

199<smiles>CCCC(C)CC</smiles>

200<smiles>CC(C)C(C)C(C)C</smiles>

201

$202 \mathrm{R}_{1}=\mathrm{O}-\mathrm{C}_{16} \mathrm{H}_{32} \mathrm{O}_{2}, \mathrm{R}_{2}=\mathrm{O}=$ $203 \mathrm{R}_{1}=\mathrm{O}-\mathrm{C}_{16} \mathrm{H}_{32} \mathrm{O}_{2}, \mathrm{R}_{2}=\mathrm{OH}$ $204 \mathrm{R}_{1}=\mathrm{OCHO}, \quad \mathrm{R}_{2}=\mathrm{H}, \mathrm{H}$ $205 \mathrm{R}_{1}=\mathrm{OH}, \quad \mathrm{R}_{2}=\mathrm{O}=$ $206 \mathrm{R}_{1}=\mathrm{OH}, \quad \mathrm{R}_{2}=\mathrm{OH}$

Figure 13. Cont. 

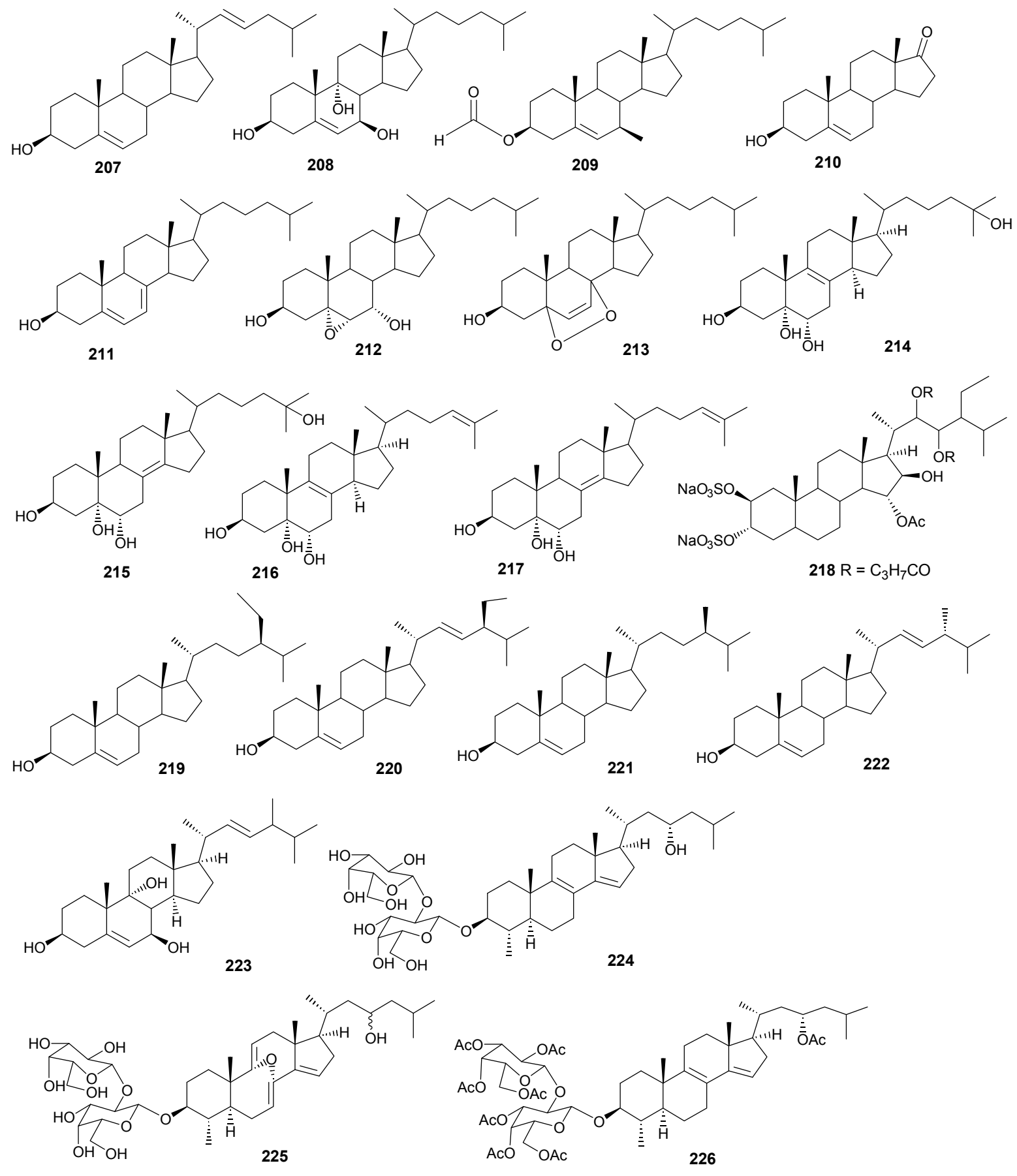

Figure 13. Steriod structures (178-226).

Moderate growth inhibition for a human colon tumor cell line was observed with 180 [58]. Compounds 184 and 186 exhibited activity against three human tumor cell lines including the lung non-small cell line A549, the glioblastoma line U373 and the prostate line PC-3 [59]. Compound 187 showed an $\mathrm{IC}_{50}$ of 6.1 and $8.2 \mu \mathrm{g} / \mathrm{mL}$ against the human cancer cell lines HepG2 and HCT, respectively [24].

Compounds 204-207 show antibacterial activity against Gram-positive (Bacillus subtilis) and Gram-negative bacteria (Pseudomonas aeruginosa), at a $1 \mu \mathrm{g} / \mathrm{ml}$ concentration [64]. Compounds 208-210 exhibited antitumor activity based on four cancer panels: HepG2, WI 38, VERO, and MCF-7 [65]. Compounds 215-220 exhibited cytotoxic effects in the tumor cell lines, HepG2 and 
MCF-7 with $\mathrm{IC}_{50}$ in the range of 20-500 $\mu \mathrm{M}$. Interestingly, 217 showed the highest affinity to DNA with IC50 $30 \mu \mathrm{g} / \mathrm{mL}$ [66]. Compounds 223 and 224 showed antifungal activity against Candida tropicalis, with petri dish inhibition at $10 \mu \mathrm{g} / \mathrm{disc}$ [67].

\section{Drug Leads}

Even though terpenes are the largest group of natural products with over 25,000 structures thus far reported, a small subset of these metabolites have been investigated for biological function and/or activity. Basic biological constituents such as membrane components, hormones, antioxidants and chemical defenses require the isoprenoid building module. Future chemical studies of marine organisms are expected to generate an ensemble of novel terpenes based on progressive knowledge on enzymatic machinery and selective pressures under which such organisms have evolved. The expanding chemo-diversity of marine terpenes is being assisted in part by advanced analytical chemistry methods for structure determination and sophisticated diving techniques for sample collection.

Methods for assaying for in vitro biological activity can be more variable in terms of stardardized protocols. The same positive or native chemical controls are not always utilized making direct comparisons of biological activity between different testing laboratories unreliable or at least not reproducible. Moreover, with the paucity of ethnomedical knowledge from marine sources, the basis for selecting the most promising bioassay can be more of an art than a science. The screening for anti-cancer activity in facilities such as the National Cancer Institute (NCI) Chemotherapeutic Agents Repository operated by Fisher BioServices [71] can provide invaluable, cost-free, sensitive screening of hits against multiple-target 60 cancer cell line panels, broadening the opportunity to conduct more comprehensive and mechanistic studies. In the case where a set of metabolites has already been identified possessing a given biological activity, computational, in silico, and pharmacophore modeling can guide future design of druggable analogues with better biological activity, without expected toxicity, even if the structural characterization of the biological target(s) is/are not feasible. Such virtual models utilizes steric and electronic descriptors to identify pharmacophoric features such as hydrophobic centroids, aromatic rings, hydrogen bonding acceptors/donors and cation/anion interactions to match optimal supramolecular interactions with a specific biological target that triggers or blocks a response. Functional group properties can also be identified for the rational semi-synthetic design of biologically active marine natural scaffolds. Strategies such as the Topliss scheme designate a series of substituents based on lipophilic, electronic and steric properties to generate multiple analogues with slight controlled chemical property differences that can be used for comprehensive structure-activity studies to obtain superior biological activity relative to the parent natural product. While these techniques and tools are not distinct or exclusive for exploring marine sources and marinederived natural products, such methods can be effective for enhancing biological activity. For example, sipholenol $\mathrm{A}$ is a noteworthy example of developing a marine metabolite using medicinal chemistry approaches to generate biologically active analogue libraries [49]. These natural product examples with exceptional biological potency outcomes $\left(\mathrm{IC}_{50}\right.$ in the low $\mu \mathrm{M}$ range for invasive breast cancer) demonstrate the potential of marine natural products for the discovery of future novel druggable entities useful for the control and management of human diseases. 


\section{Conclusions}

Terpenoids provide a vast array of molecular architectures with the coral community of the Red Sea having added significantly to the structure database over the last thirty years. While marine invertebrates in this ecosystem are still being discovered, interest in both the chemistry and biological activity of Red Sea terpenes has generated many novel structures with promising biological activities.

\section{Conflicts of Interest}

The authors declare no conflict of interest.

\section{References}

1. McCarthy, P.J.; Pomponi, S.A. A search for new pharmaceutical drugs from marine organisms. Mar. Biomed. Res. 2004, 22, 1-2.

2. Appeltans, W.; Ahyong, S.T.; Anderson, G.; Angel, M.V.; Artois, T.; Bailly, N.; Bamber, R.; Barber, A.; Bartsch, I.; Berta, A.; et al. The magnitude of global marine species diversity. Curr. Biol. 2012, 22, 2189-2202.

3. Kinghorn, A.D.; Pan, L.; Fletcher, J.N.; Chai, H. The relevance of higher plants in lead compound discovery programs. J. Nat. Prod. 2011, 74, 1539-1555.

4. Fuesetani, N. In Drugs from the Sea; Fuesetani, M., Ed.; Karger: Basel, Switzerland, 2000; Chapter 1, pp. 1-5.

5. Faulkner, D. Biomedical uses for natural marine chemicals. J. Ocean. 1992, 35, 29-35.

6. Edwards, A.J.; Head, S.M. Key Environments-Red Sea; Pergamon Press: Oxford, UK, 1987; p. 440.

7. Tursch, B.; Tursch, A. The soft coral community on a sheltered reef quadrat at Laing Island (Papua New Gunea). Mar. Biol. 1982, 68, 321-332.

8. McFadden, C.S.; Sánchez, J.A.; France, S.C. Molecular phylogenetic insights into the evolution of Octocorallia: A review. Int. Comp. Biol. 2010, 50, 389-410.

9. Elkhayat, E.S.; Ibrahim, S.R.M.; Fouad, M.A.; Mohamed, G.A. Dendronephthols A-C, new sesquiterpenoids from the Red Sea soft coral Dendronephthya sp. Tetrahedron 2014, 70, 3822-3825.

10. El Sayed, K.A.; Hamann, M.T. A new norcembranoid dimer from the Red Sea soft coral Sinularia gardineri. J. Nat. Prod. 1996, 59, 687-689.

11. Ellithey, M.S.; Lall, N.; Hussein, A.A.; Meyer, D. Cytotoxic, Cytostatic and HIV-1 PR inhibitory activities of the soft coral Litophyton arboretum. Mar. Drugs 2013, 11, 4917-4936.

12. Al-Footy, K.O.; Alarif, W.M.; Asiri, F.; Aly, M.M.; Ayyad, S.N. Rare pyrane-based cembranoids from the Red Sea soft coral Sarcophyton trocheliophorum as potential antimicrobial antitumor agents. Med. Chem. Res. 2015, 24, 505-512.

13. Al-Lihaibi, S.S.; Alarif, W.M.; Abdel-Lateff, A.; Ayyad, S.N.; Abdel-Naim, A.B.; El-Senduny, F.F.; Badria, F.A. Three new cembranoid-type diterpenes from Red Sea soft coral Sarcophyton glaucum: Isolation and anti-proliferative activity against HepG2 cells. Eur. J. Med. Chem. 2014, 81, 314-322. 
14. Kelman, D.; Benayahu, Y.; Kashman, Y. Variation in secondary metabolite concentration in yellow and grey morphs of the red sea soft coral Parerythropodium fulvum fulvum: Possible ecological implications. J. Chem. Ecol. 2000, 26, 1123-1133.

15. Youssef, D.T.A.; Yoshida, W.Y.; Kelly, M.; Scheuer, P.J. Cytotoxic cyclic norterpene peroxides from a Red Sea sponge Diacarnus erythraenus. J. Nat. Prod. 2001, 64, 1332-1335.

16. Youssef, D.T.A.; Singab, A.B.; van Soest, R.W.M.; Fusetani, N. Hyrtiosenolides A and B, two new sesquiterpene-methoxybutenolides and a new sterol from a Red Sea sponge Hyrtios species. J. Nat. Prod. 2004, 67, 1736-1739.

17. Shaaban, M.; Shaaban, K.A.; Ghani, M.A. Hurgadacin: A new steroid from Sinularia polydactyla. Steroids 2013, 78, 866-873.

18. Kondracki, M.B.; Guyot, M. A New Sesquiterpene tetronic acid derivative from the marine sponge Smenospongia sp. Tetrahedron Lett. 1999, 40, 3149-3150.

19. Abad, M.J.; Berimejo, P. Bioactive natural products from marine sources. Stud. Nat. Prod. Chem. 2001, 25, 683-755.

20. Hirsch, S.; Rudi, A.; Kashman, Y.; Loya, Y. New avarone and avarol derivatives from the marine sponge Dysidea cinerea. J. Nat. Prod. 1991, 54, 92-97.

21. Elkhateeb, A.; El-Beih, A.A.; Gamal-Eldeen, A.M.; Alhammady, M.A.; Ohta, S.; Paré, P.W.; Hegazy, M.F. New terpenes from the Egyptian soft coral Sarcophyton Ehrenberg. Mar. Drugs 2014, 12, 1977-1986.

22. Abou El-Ezz, R.F.; Ahmed, S.A.; Radwan, M.M.; Ayoub, N.A.; Afifi, M.S.; Ross, S.A.; Szymanski, P.T.; Fahmy, H.; Khalifa, S.I. Bioactive cembranoids from the Red Sea soft coral Sarcophyton glaucum. Tetrahedron Lett. 2013, 54, 989-992.

23. Hegazy, M.F.; Mohameda, T.A.; Abdel-Latif, F.F.; Alsaid, M.S.; Shahat, A.A.; Paré, P.W. Trochelioid A and B, new cembranoid diterpenes from the Red Sea soft coral Sarcophyton trocheliophorum. Phytochem. Lett. 2013, 6, 383-386.

24. Aboutabl, E.A.; Azzam, S.M.; Michel, C.G.; Selim, N.M.; Hegazy, M.F.; Ali, A.A.M.; Hussein, A.A. Bioactive terpenoids from the Red Sea soft coral Sinularia polydactyla. Nat. Prod. Res. 2013, 27 , 2224-2226.

25. Hegazy, M.F.; Gamal Eldeen, A.M.; Shahat, A.A.; Abdel-Latif, F.F.; Mohamed, T.A.; Whittlesey, B.R.; Paré, P.W. Bioactive hydroperoxyl cembranoids from the Red Sea soft coral Sarcophyton glaucum. Mar. Drugs 2012, 10, 209-222.

26. Hegazy, M.F.; El-Beihb, A.A.; Moustafad, A.Y.; Hamdy, A.A.; Alhammady, M.A.; Selim, R.M.; Abdel-Rehimb, M.; Paré, P.W. Cytotoxic cembranoids from the Red Sea soft coral Sarcophyton glaucum. Nat. Prod. Commun. 2011, 6, 1809-1812.

27. Kinamoni, Z.; Groweiss, A.; Carmely, S.; Kashman, Y.; Loya, Y. Several new cembranoid diterpenes from three soft coral of the Red Sea. Tetrahedron 1983, 39, 1641-1948.

28. Groweiss, A.; Kashman, Y. Eight new xenia diterpenoids from three soft coral of the Red Sea. Tetrahedron 1983, 39, 3385-3396.

29. Hassan, H.M.; Khanfar, M.A.; Elnagar, A.Y.; Mohammed, R.; Shaala, L.A.; Youssef, D.T.A.; Hifnawy, M.S.; El Sayed, K.A. Pachycladins A-E, prostate cancer invasion and migration inhibitory eunicellin-based diterpenoids from the Red Sea soft coral Cladiella pachyclados. J. Nat. Prod. 2010, 73, 848-853. 
30. Badria, F.A.; Guirguis, A.N.; Perovic, S.; Steffen, R.; Müller, W.E.; Schröder, H.C. Sarcophytolide: A new neuroprotective compound from the soft coral Sarcophyton glaucum. Toxicology. 1998, 131, 133-143.

31. Eltahawy, N.A.; Ibrahim, A.K.; Radwan, M.M.; ElSohly, M.A.; Hassanean, H.A.; Ahmed, S.A. Cytotoxic cembranoids from the Red Sea soft coral, Sarcophyton auritum. Tetrahedron Lett. 2014, 55, 3984-3988.

32. Isaacs, S.; Kashman, Y. Juncins A-F, six new briarane diterpenoids from Gorogonian Junceella Juncea. J. Nat. Prod. 1990, 53, 596-602.

33. Wiemer, D.F.; Meinwald, J.; Prestwich, G.D.; Solheim, B.A.; Clardy, J. Biflora-4,10(19),15-triene: A new diterpene from a termite soldier (Isoptera Termitidae Termitinae). J. Org. Chem. 1980, 45, 191-192.

34. Rudi, A.; Kashman, Y. Chelodane, barekoxids, and Zaatirin-three new diterpenoids from the marine sponge chelonaplysilla erecta. J. Nat. Prod. 1992, 55, 1408-1414.

35. Rudi, A.; Kshman, Y. Three new norrisolide related rearranged spongians. Tetrahedron 1990, 64, 4019-4022.

36. Youssef, D.T.A.; Shaala, L.A.; Emara, S. Antimycobacterial scalarane-based sesterterpenes from the Red Sea sponge Hyrtios erecta. J. Nat. Prod. 2005, 68, 1782-1784.

37. Youssef, D.T.A.; Yamaki, R.K.; Kelly, M.; Scheuer, P.J. Salmahyrtisol A, a novel cytotoxic sesterterpene from the Red Sea sponge Hyrtios erecta. J. Nat. Prod. 2002, 65, 2-6.

38. Ashour, M.A.; Elkhayat, E.S.; Ebel, R.; Edrada, R.; Proksch, P. Indole alkaloid from the Red Sea sponge Hyrtios erectus. ARKIVOC 2007, 15, 225-231.

39. Fontana, A.; Fakhr, I.; Mollo, E.; Cimino, G. (-)-Wistarin from the marine sponge Ircinia sp.: The first case of enantiomeric sesterterpenes. Tetrahedron Asymmetry 1999, 10, 3869-3872.

40. Rudi, A.; Yosief, T.; Schleyer, M.; Kashman, Y. Bilosespens A and B: Two novel cytotoxic sesterpenes from the Marine Sponge Dysidea cinerea. Org. Lett. 1999, 3, 471-472.

41. Lefranc, F.; Nuzzo, G.; Hamdy, N.A.; Fakhr, I.; Banuls, L.M.Y.; Van Goietsenoven, G.; Villani, G.; Mathieu, V.; van Soest, R.; Kiss, R.; et al. In vitro pharmacological and toxicological effects of norterpene peroxides isolated from the Red Sea sponge Diacarnus erythraeanus on normal and cancer cells. Nat. Prod. 2013, 76, 1541-1547.

42. Youssef, D.T.A. Tasnemoxides A-C, new cytotoxic cyclic norsesterterpene peroxides from the Red Sea sponge Diacarnus erythraenus. J. Nat. Prod. 2004, 67, 112-114.

43. Kashman, Y.; Rotem, M. Muqubilin, a new C24-Isoprenoid from a marine sponge. Tetrahedron Lett. 1979, 20, 1707-1708..

44. Guo, Y.; Gavagnin, M.; Mollo, E.; Trivellone, E.; Cimino, G. A new norsesterterpene peroxide from a red sea sponge. Nat. Prod. Lett. 1996, 9, 105-112.

45. El Sayed, K.A.; Hamann, M.T.; Hashish, N.E.; Shier, W.T.; Kelly, M.; Khan, A.A. Antimalarial, antiviral, and antitoxoplasmosis norsesterterpene peroxide acids from the Red Sea sponge Diacarnus erythraeanus. J. Nat. Prod. 2001, 64, 522-524.

46. Carmely, S.; Kashman, Y. Neviotine-A, A new triterpene from the Red Sea sponge Siphonochalina siphonella. J. Org. Chem. 1986, 51, 784-788. 
47. Ayyad, S.N.; Angawi, R.F.; Saqer, E.; Abdel-Lateff, A.; Badria, F.A. Cytotoxic neviotane triterpene-type from the red sea sponge Siphonochalina siphonella. Pharmacogn. Mag. 2014, 10, 334-341.

48. Abraham, I.; Jain, S.; Wuc, C.P.; Khanfar, M.A.; Dai, Y.K.C.; Shi, Z.; Chen, X.; Fu, L.; Ambudkar, S.V.; El Sayed, K.; et al. Marine sponge-derived sipholane triterpenoids reverse P-glycoprotein (ABCB1)-mediated multidrug resistance in cancer cells. Biochem. Pharmacol. 2010, 80, 1497-1506

49. Akl, M.R.; Foudah, A.I.; Ebrahim, H.Y.; Meyer, S.A.; El Sayed, K.A. The marine-derived sipholenol A-4-O-3',4'-dichlorobenzoate inhibits breast cancer growth and motility in vitro and in vivo through the suppression of Brk and FAK signaling. Mar. Drugs 2014, 12, 2282-2304.

50. Foudah, A.I.; Sallam, A.A.; Akl, M.R.; El Sayed, K.A. Optimization, pharmacophore modeling and 3D-QSAR studies of sipholanes as breast cancer migration and proliferation inhibitors. Eur. J. Med. Chem. 2014, 73, 310-324.

51. Jain, S.; Abraham, I.; Carvalho, P.; Kuang, Y.H.; Shaala, L.A.; Youssef, D.T.A.; Avery, M.A.; El Sayed, K.A. Sipholane triterpenoids: Chemistry, reversal of ABCB1/P-Glycoprotein-mediated multidrug resistance, and pharmacophore modeling. J. Nat. Prod. 2009, 72, 1291-1298.

52. Ayyad, S.N.; Angawi, R.F.; Alarif, W.M.; Saqera, E.A.; Badriae, F.A. Cytotoxic Polyacetylenes from the Red Sea Sponge Siphonochalina siphonella. Z. Naturforschung C 2014, 69, 117-123.

53. Shi, Z.; Jain, S.; Kim, I.W.; Peng, X.X.; Abraham, I.; Youssef, D.T.A.; Fu, L.W.; Sayed, K.E.; Ambudkar, S.V.; Chen, Z.S. Sipholenol A, a marine-derived sipholane triterpene, potently reverses P-glycoprotein (ABCB1)-mediated multidrug resistance in cancer cells. Cancer Sci. 2007, 98, 1373-1380.

54. Isaacs, S.; Kashman, Y. Shaagrockol B and C: Two hexaprenylhydroquinone disulfates from the Red Sea sponge Toxiclona toxius. Tetrahedron Lett. 1992, 33, 2227-2230.

55. Kashman, Y.; Yosief, T.; Carmeli, S. New triterpenoids from the Red Sea sponge Siphonochalina siphonella. J. Nat. Prod. 2001, 64, 175-180.

56. Jain, S.; Laphookhieo, S.; Shi, Z.; Fu, L.; Akiyama, S.; Chen, Z.S.; Youssef, D.T.A.; Soest, R.W.M.; El Sayed, K.A. Reversal of P-glycoprotein-mediated multidrug resistance by sipholane triterpenoids. J. Nat. Prod. 2007, 70, 928-931.

57. Ahmed, S.; Ibrahim, A.; Arafa, A. Anti-H5N1 virus metabolites from the Red Sea soft coral Sinularia candidula. Tetrahedron Lett. 2013, 54, 2377-2381.

58. Elshamy, A.I.; Abdel-Razik, A.F.; Nassar, M.I.; Mohamed, T.K.; Ibrahim, M.A.; El-Kousy, S.M. A new gorgostane derivative from the Egyptian Red Sea soft coral Heteroxenia ghardaqensis. Nat. Prod. Res. 2013, 27, 1250-1254.

59. Mohamed, G.A.; Abd-Elrazek, A.E.E.; Hassanea, H.A.; Youssef, D.T.A.; van Soes, R. New compounds from the Red Sea marine sponge Echinoclathria gibbosa. Phytochemistry Lett. 2014, 9, 51-58.

60. Carmely, S.; Kashman, Y. Isolation and structure elucidation of Lobophytosterol and three other closely related sterols: Five new $\mathrm{C}_{28}$ polyoxygenated sterols from the red sea soft coral lobophytum depressum. Tetrahedron 1981, 37, 2397-2403.

61. Goob, R.; Rudi, A.; Kshman, Y. Three new glycolipids from a Red Sea sponge of the genus Erylus. Tetrahedron 1996, 52, 7921-7928. 
62. Fouad, M.; Al-Trabeen, K.; Badran, M.; Wray, V.; Edrada, R.; Proksch, P.; Ebela, R. New steroidal saponins from the sponge Erylus lendenfeldi. ARKIVOC 2004, 13, 17-27.

63. Carmely, S.; Michal, R.; Loya, Y.; Kashman, Y. The structure of eryloside A, a new antitumor and anti fungal 4-methylated steroidal glycoside from the sponge Erylus lendenfeldi. J. Nat. Prod. 1989, 52, 167-170.

64. Al-lihaibi, S.S.; Ayyad, S.N.; Shaher, F.; Alarif, W.M. Antibacterial sphingolipid and steroids from the black coral Antipathes dichotoma. Chem. Pharm. Bull. 2010, 58, 1635-1638.

65. Alarif, W.M.; Abdel-lateff, A.; AL-lihaibi, S.S.; Ayyad, S.N.; Badria, F.A.; Alsofyani, A.A.; Abou-Elnaga, Z.S. Marine bioactive steryl esters from the Red Sea black coral Antipathes dichotoma. Clean Soil Air Water 2012, 41, 1116-1121.

66. Abdel-lateff, A.; Alarif, W.M.; Asfour, H.Z.; Ayyad, S.N.; Khedr, A.; Badria, F.A.; Al-lihaibi, S.S. Cytotoxic effects of three new metabolites from Red Sea marine sponge, Petrosia sp. Environ. Toxicol. Pharmacol. 2014, 37, 928-935.

67. Sauleau, P.; Bourguet-Kondracki, M.L. Novel polyhydroxysterols from the Red Sea marine sponge Lamellodysidea herbacea. Steroids 2005, 70, 954-959.

68. Rudi, A.; Yosief, T.; Loya, S.; Hizi, A.; Schleyer, M.; Kashman, Y. Clathsterol, a Novel Anti-HIV-1 RT Sulfated Sterol from the sponge Clathria species. J. Nat. Prod. 2001, 64, 1451-1453.

69. Abou-Hussein, D.R.; Badr, J.M.; Youssef, D.T.A. Dragmacidoside: A new nucleoside from the Red Sea sponge Dragmacidon coccinea. Nat. Prod. Res. 2014, 28, 1134-1141.

70. Shaaban, M.; Shaaban, K.A.; Abd-Alla, H.I.; Hanna, A.G.; Laatsch, H. Dendrophen, a novel glycyrrhetyl amino acid from Dendronephthya hemprichi. Z. Naturforschung 2011, 66, 425-432.

71. Fisher BioServices, Available online: http://www.fisherbioservices.com/company (accessed on 11 May 2015).

(C) 2015 by the authors; licensee MDPI, Basel, Switzerland. This article is an open access article distributed under the terms and conditions of the Creative Commons Attribution license (http://creativecommons.org/licenses/by/4.0/). 